\title{
Decentralised sliding mode control for a class of nonlinear interconnected systems by static state feedback
}

\author{
Jiehua Feng ${ }^{1}$, Dongya Zhao ${ }^{1 *}$, Xing-Gang Yan $^{2}$, Sarah K. Spurgeon ${ }^{3,1}$ \\ 1. College of New Energy, China University of Petroleum (East China), Qingdao, China, 266580; \\ 2. School of Engineering \& Digital Arts, University of Kent, United Kingdom, Canterbury CT2 7NT; \\ 3. Department of Electronic \& Electrical Engineering, University College London, Torrington Place, London \\ WC1E 7JE, U. K. \\ * Corresponding author’s Email: dyzhao@upc.edu.cn
}

\begin{abstract}
In this paper, a class of interconnected systems is considered, where the nominal isolated systems are fully nonlinear. A robust decentralised sliding mode control based on static state feedback is developed. By local coordinate transformation and feedback linearization, the interconnected system is transformed to a new regular form. A composite sliding surface which is a function of the system state variables is proposed and the stability of the corresponding sliding mode dynamics is analysed. A new reachability condition is proposed and a robust decentralised sliding mode control is then designed to drive the system states to the sliding surface in finite time and maintain a sliding motion thereafter. Both uncertainties and interconnections are allowed to be unmatched and are assumed to be bounded by nonlinear functions. The bounds on the uncertainties and interconnections have more general forms when compared with existing work. A MATLAB simulation example is used to demonstrate the effectiveness of the proposed method.
\end{abstract}

Key Words: Decentralised control, Sliding mode control, Nonlinear interconnected systems, Static state feedback.

\section{INTRODUCTION}

Increasingly complex industrial requirements coupled with rapid technological developments are producing engineering systems that are interconnected to form large-scale interconnected systems [1]. Such systems exist widely in multiple domains including process networks, power grids, offshore platforms, urban traffic networks as well as ecosystems [2]. Large-scale complex systems can require high on-line computing power to implement controllers. For example, in a centralized control implementation, all of the system information may be used to control the interconnected system. Such a large computational burden may not be attractive to industry as data transfer requirements are large and risks of cyber attack increase. Plant shut down may result from corruption or blockage of data transfer and the system robustness may be compromised [3]. This has motivated the development of decentralised control strategies in which only local subsystem information is needed for local control design. A decentralized control approach has the potential to improve the computational efficiency and the overall safety of the system. Hence the study of decentralized control is of significant importance for interconnected systems [4].

Decentralized control has been studied for many years and many results have been obtained relating to interconnected systems [5], [6], [7]. It is well known that uncertainties or modeling errors can seriously affect the performance of controlled systems. The uncertainties in subsystems will not only affect their own performance, but also affect the entire system's performance through the interactions among subsystems. In addition, the coupling between subsystems also brings difficulties to the stabilization of interconnected large-scale systems. Robust control schemes and adaptive control schemes are often designed to reject or reduce the effect of the uncertainties caused by the modeling errors within subsystems and the coupling among subsystems. Adaptive control can only be applied to some special systems, i.e. parametric uncertainties satisfying linear growth conditions [8], [9]. Alternative robust control methods, such as $H_{\infty}$ control [10], [11], have strong robustness in dealing with large-scale operating regions even in the presence of disturbance scenarios, but the design process is complex and the synthesis can be difficult and time-consuming [12], [13].

As one of the classical robust control methods, sliding mode control [14] is widely considered to be an effective method to deal with uncertainties due to its excellent performance characteristics and relatively simple design process. Decentralized 
sliding mode control has been extensively studied in large-scale systems. Based on state information, a decentralized sliding mode control is designed to deal with the matched uncertainties in [15]. A decentralised adaptive sliding mode control is designed for large-scale systems with unmatched perturbations in [16]. A type of decentralised output feedback sliding mode control is designed for a class of interconnected systems in [17], where only matched uncertainties and interconnections are considered. A class of interconnected systems is stabilized by an output feedback sliding mode controller with a more general structure for the interconnections and unmatched uncertainties in [18]. Note that these contributions require that the nominal isolated systems are linear. In practice, almost all systems are nonlinear and have nonlinear behaviour [19]. Moreover, the majority of control schemes for linear systems cannot be applied to nonlinear systems directly. This motivates the current study of nonlinear interconnected systems with nonlinear nominal isolated systems and nonlinear interconnections.

Recently, the study of interconnected systems with nonlinear nominal isolated systems has received great attention and some interesting results have been achieved. Based on static output feedback, decentralized stabilization of nonlinear interconnected systems has been achieved in [20]. However, this output feedback method imposes higher requirements for the system model. The decentralized tracking control problem is studied for a class of interconnected stochastic nonlinear systems in [21], where the result obtained can only guarantee that the closed-loop system is practically stable. A smooth dynamic output feedback control is proposed for the case of multiple time-delay systems with nonlinear uncertainties in [22]. However, it is based on dynamic feedback, which increases the system dimension as well as the computational complexity. A decentralised state feedback control scheme is proposed for a class of time delay interconnected systems with matched interconnections, and a new term, called weak unmatched uncertainty, is introduced in [23]. It should be noted that high control gains are used in [23] to reject the effect of weak unmatched uncertainties and the matched interconnections, which in turn may lead to control input saturation. An adaptive dynamic programming technique is introduced for nonlinear large-scale systems in [24]. Although the nominal isolated systems are nonlinear, only matched interconnections are considered. A multi-dimensional Taylor network decentralised control is investigated for large-scale stochastic nonlinear systems in [25], but only the tracking of specific signals has been considered. The knowledge that sliding mode control can accommodate unmatched uncertainties [26] provides further motivation for considering decentralised sliding mode control for nonlinear interconnected systems.

There are few results which apply sliding mode control methods to interconnected systems with nonlinear nominal isolated systems. A decentralised control strategy based on sliding mode techniques is proposed for a class of nonlinear interconnected systems in regular form in [27]. Although this method does not require the nominal isolated subsystems to be linearized or partially linearized, it is assumed that the system is in regular form, which requires transformation of the nonlinear systems; a constructive method to obtain the transformation matrix is not generally available. Further, the proposed control does not guarantee that the system states reach the sliding surface in finite time.

In this paper, a decentralised static sliding mode control strategy is proposed for a class of nonlinear interconnected systems. It is not required that the nominal isolated systems are linear or linearizable. Under the conditions that all the nominal isolated subsystems have uniform relative degree and the distribution is involutive, the class of nonlinear interconnected systems considered can be transformed to a convenient regular form representation using a local coordinate transformation and feedback linearization. In comparison with most of the existing control methods for interconnected systems [15], [16], [28], the nominal isolated subsystems of the interconnected system considered in this paper are fully nonlinear, which extends both the potential practical application value and theoretical significance. The proposed regular form greatly facilitates decentralised control design. Given the regular form, a decentralized sliding mode control can be designed to accommodate both matched and unmatched uncertainties. A set of sufficient conditions is developed to guarantee that the corresponding sliding motion is asymptotically stable using a Lyapunov approach, where the conservativeness of the interconnection terms is reduced compared with the results in [23], [27]. Meanwhile a new reachability condition is proposed for the nonlinear interconnected system which forces the system states to reach the sliding surface in finite time; the existing reachability frameworks available in the literature cannot guarantee this. The main theoretical contributions of this paper are: (i) a framework is proposed to transform the nonlinear interconnected system into a new regular form; (ii) a set of sufficient conditions is given to guarantee the largescale interconnected system has an asymptotically stable sliding motion; (iii) a new reachability condition is proposed so that the decentralised sliding mode control forces the system to reach the composite sliding surface in finite time. 
The remainder of the paper is organized as follows. Section 2 formulates the problem and gives some definitions that will be used in the following sections. In Section 3, basic assumptions and stability analysis of the sliding mode are given. In Section 4, a decentralised sliding mode control is designed. In Section 5, a simulation example is presented to validate the proposed approach. Conclusions are given in Section 6.

\section{INTERCONNECTED SYSTEM ANALYSIS AND PROBLEM FORMULATION}

Consider the nonlinear interconnected system described by:

$$
\dot{x}_{i}=f_{i}\left(x_{i}\right)+g_{i}\left(x_{i}\right)\left(u_{i}+\phi_{i}\left(t, x_{i}\right)\right)+\xi_{i}\left(t, x_{i}\right)+\sum_{j=1}^{n} H_{i j}\left(t, x_{j}\right), i=1,2, \cdots n
$$

where $x_{i} \in \chi_{i} \in R^{n_{i}}, u_{i} \in R^{m_{i}}$ are the state and input of the $i$-th subsystem respectively, $\chi_{i}$ is a neighborhood of the origin and $x:=\operatorname{col}\left(x_{1}, \ldots, x_{n}\right) \in \chi:=\chi_{1} \times \cdots \times \chi_{n}, f_{i}\left(x_{i}\right):=\left[f_{i 1}\left(x_{i}\right), \ldots, f_{i n_{i}}\left(x_{i}\right)\right]^{T} \in R^{n_{i}}, g_{i}\left(x_{i}\right):=\left[g_{i 1}\left(x_{i}\right), \ldots, g_{i m_{i}}\left(x_{i}\right)\right] \in$ $R^{n_{i} \times m_{i}}$ is the input distribution matrix, $\phi_{i}\left(t, x_{i}\right) \in R^{m_{i}}$ and $\xi_{i}\left(t, x_{i}\right) \in R^{n_{i}}$ represent the matched uncertainties and unmatched uncertainties respectively, $\sum_{j=1}^{n} H_{i j}\left(t, x_{j}\right)$ denotes the unknown interconnections.

The following definitions are provided for ease of exposition.

Definition 1: [20] The system

$$
\dot{x}_{i}=f_{i}\left(x_{i}\right)+g_{i}\left(x_{i}\right)\left(u_{i}+\phi_{i}\left(t, x_{i}\right)\right)+\xi_{i}\left(t, x_{i}\right), \quad i=1,2, \cdots n
$$

is referred to as the $i$-th isolated subsystem of the interconnected system (1), and the system

$$
\dot{x}_{i}=f_{i}\left(x_{i}\right)+g_{i}\left(x_{i}\right) u_{i}, \quad i=1,2, \cdots n
$$

is referred to as the $i$-th nominal isolated subsystem of the interconnected system (1).

Definition 2: [29] System (3) is said to have uniform relative degree $\left(r_{i 1}, r_{i 2}, \ldots, r_{i m_{i}}\right)$ in domain $\chi_{i}$ if there exists a series of functions $h_{i j}\left(x_{i}\right) \in R^{m_{i}}$ such that for any $x_{i} \in \chi_{i}$ :

i) $L_{g_{i j}} L_{f_{i}}^{k_{i}} h_{i l}\left(x_{i}\right)=0$ for all $1 \leqslant j \leqslant m_{i}, \quad 1 \leqslant l \leqslant m_{i}, 0 \leqslant k<r_{i}-1$ and $i=1,2, \cdots n$.

ii) A series of functions $\Lambda_{i}\left(x_{i}\right) \in R^{m_{i} \times m_{i}}$ are nonsingular for $i=1,2, \cdots n$. where

$$
\Lambda_{i}\left(x_{i}\right)=\left[\begin{array}{ccc}
L_{g_{i 1}} L_{f_{i}}^{r_{i 1}-1} h_{i 1}\left(x_{i}\right) & \cdots & L_{g_{i m_{i}}} L_{f_{i}}^{r_{i 1}-1} h_{i 1}\left(x_{i}\right) \\
L_{g_{i 1}} L_{f_{i}}^{r_{i 2}-1} h_{i 2}\left(x_{i}\right) & \cdots & L_{g_{i m_{i}}} L_{f_{i}}^{r_{i 2}-1} h_{i 2}\left(x_{i}\right) \\
\cdots & \ldots & \cdots \\
L_{g_{i 1}} L_{f_{i}}^{r_{i m_{i}}-1} h_{i m_{i}}\left(x_{i}\right) & \cdots & L_{g_{i m_{i}}} L_{f_{i}}^{r_{i m_{i}}-1} h_{i m_{i}}\left(x_{i}\right)
\end{array}\right]
$$

where $L_{f_{i}} h_{i l}\left(x_{i}\right)$ denotes the derivative of $h_{i l}\left(x_{i}\right)$ along $f_{i}$ defined by $L_{f_{i}} h_{i l}\left(x_{i}\right):=\sum_{s=1}^{n_{i}} \frac{\partial h_{i l}}{\partial x} f_{i s}(x)$ and $L_{f_{i}}^{k} h_{i l}\left(x_{i}\right)$ represents a recursion defined by $L_{f_{i}}^{k} h_{i l}\left(x_{i}\right):=\frac{\partial L_{f_{i}}^{k-1} h_{i l}\left(x_{i}\right)}{\partial x} f_{i}(x)$ with $L_{f_{i}}^{0} h_{i l}\left(x_{i}\right):=h_{i l}\left(x_{i}\right)$.

Remark 1: Note that $r_{i}=\sum_{l=1}^{m_{i}} r_{i l} \leqslant n_{i}$. When $r_{i}=n_{i}$, system (3) can be linearized completely for $i=1,2, \cdots n$, and in this case, there is no zero dynamics. When $r_{i}<n_{i}$, system (3) can only be partially feedback linearized, and in this case, there are nonlinear zero dynamics. It should be pointed out that it is not required that $r_{i}=n_{i}$ in this paper and thus it does not require that the nominal isolated subsystems are linearizable.

For simplicity, the distribution formed by the vector fields $g_{i 1}\left(x_{i}\right), g_{i 2}\left(x_{i}\right), \ldots, g_{i m_{i}}\left(x_{i}\right)$ is denoted by:

$$
G_{i}\left(x_{i}\right):=\operatorname{span}\left\{g_{i 1}\left(x_{i}\right), g_{i 2}\left(x_{i}\right), \ldots, g_{i m_{i}}\left(x_{i}\right)\right\}
$$

In this paper, it is assumed that the $i$-th nominal isolated subsystem of the interconnected system (1) has uniform relative degree $\left(r_{i 1}, r_{i 2}, \ldots, r_{i m_{i}}\right)$ and the distribution $G_{i}\left(x_{i}\right)$ is involutive in domain $\chi_{i}$ for $i=1,2, \cdots n$. For the convenience of analysis and design, the feedback linearization technique is applied to the system (3). 
Let $z_{i l}=\left[\begin{array}{llll}h_{i l}\left(x_{i}\right) & L_{f_{i}} h_{i l}\left(x_{i}\right) & \cdots & L_{f_{i}}^{r_{i l}-1} h_{i l}\left(x_{i}\right)\end{array}\right]^{T}, l=1,2, \ldots, m_{i}$ with $z_{i l} \in R^{r_{i l}}$. From [29], there exist $n_{i}-r_{i}$ functions $z_{i\left(r_{i}+1\right)}, z_{i\left(r_{i}+2\right)}, \cdots, z_{i n_{i}}$ defined in $\chi_{i}$ such that the Jacobian matrix of the mapping

$$
T_{i}: x_{i} \rightarrow z_{i}:=\operatorname{col}\left(z_{i}^{a}, z_{i}^{b}\right)
$$

is nonsingular, where $z_{i}^{a}:=\operatorname{col}\left(z_{i 1}, z_{i 2}, \cdots, z_{i m_{i}}\right) \in R^{r_{i}}$ and $z_{i}^{b}:=\operatorname{col}\left(z_{i\left(r_{i}+1\right)}, z_{i\left(r_{i}+2\right)}, \cdots, z_{i n_{i}}\right) \in R^{n_{i}-r_{i}}$. Therefore, $T_{i}$ in (6) defines a diffeomorphism.

It follows from [29] that in the new coordinate system $z_{i}$, system (3) can be described as:

$$
\begin{aligned}
& \dot{z}_{i}^{a}=\bar{A}_{i} z_{i}^{a}+\bar{B}_{i}\left(\alpha_{i}\left(z_{i}\right)+\Lambda_{i}\left(z_{i}\right) u_{i}\right) \\
& \dot{z}_{i}^{b}=\omega_{i}\left(z_{i}^{a}, z_{i}^{b}\right)
\end{aligned}
$$

where $\bar{A}_{i}=\operatorname{diag}\left\{\bar{A}_{i 1}, \cdots, \bar{A}_{i l}, \cdots \bar{A}_{i m_{i}}\right\} \in R^{r_{i} \times r_{i}}, \bar{B}_{i}=\operatorname{diag}\left\{\bar{B}_{i 1}, \cdots, \bar{B}_{i l}, \cdots, \bar{B}_{i m_{i}}\right\} \in R^{r_{i} \times m_{i}}$,

$$
\alpha_{i}\left(z_{i}\right)=\left[\begin{array}{c}
L_{f_{i}}^{r_{i 1}} h_{i 1}\left(z_{i}\right) \\
L_{f_{i}}^{r_{i 2}} h_{i 2}\left(z_{i}\right) \\
\vdots \\
L_{f_{i}}^{r_{i m_{i}}} h_{i m_{i}}\left(z_{i}\right)
\end{array}\right] \quad \bar{A}_{i l}=\left[\begin{array}{cccccc}
0 & 1 & 0 & \vdots & 0 & 0 \\
0 & 0 & 1 & \vdots & 0 & 0 \\
\vdots & \vdots & \vdots & \ddots & \vdots & \vdots \\
0 & 0 & 0 & \cdots & 0 & 1 \\
0 & 0 & 0 & \cdots & 0 & 0
\end{array}\right]_{r_{i l} \times r_{i l}} \quad \bar{B}_{i l}=\left[\begin{array}{c}
0 \\
0 \\
\vdots \\
0 \\
1
\end{array}\right]_{r_{i l} \times 1}
$$

and $\Lambda_{i}$ is defined in (4).

Design a state feedback

$$
u_{i}=-\Lambda_{i}^{-1}\left(z_{i}\right) \alpha_{i}\left(z_{i}\right)+\Lambda_{i}^{-1}\left(z_{i}\right) \nu_{i}
$$

where $\nu_{i} \in R^{m_{i}}$ is the new input of the $i$-th subsystem for $i=1,2, \cdots n$.

By applying the feedback from (9) to system (7), the corresponding closed-loop system can be described as:

$$
\begin{aligned}
& \dot{z}_{i}^{a}=\bar{A}_{i} z_{i}^{a}+\bar{B}_{i} \nu_{i} \\
& \dot{z}_{i}^{b}=\omega_{i}\left(z_{i}^{a}, z_{i}^{b}\right)
\end{aligned}
$$

Consider the interconnected system (1). According to the above analysis, it follows from (10) that in the new coordinates $z:=\operatorname{col}\left(z_{1}, \ldots, z_{n}\right) \in \mathrm{Z}:=\mathrm{Z}_{1} \times \cdots \times \mathrm{Z}_{n}$ given in (6), system (1) can be described by:

$$
\begin{gathered}
\dot{z}_{i 1}^{a}=A_{i} z_{i 1}^{a}+B_{i} z_{i 2}^{a}+\Xi_{i 1}^{a}\left(t, z_{i}\right)+\Psi_{i 1}^{a}(t, z) \\
\dot{z}_{i}^{b}=\omega_{i}\left(z_{i 1}^{a}, z_{i 2}^{a}, z_{i}^{b}\right)+\Xi_{i}^{b}\left(t, z_{i}\right)+\Psi_{i}^{b}(t, z) \\
\dot{z}_{i 2}^{a}=\nu_{i}+\Xi_{i 2}^{a}\left(t, z_{i}\right)+\Psi_{i 2}^{a}(t, z)
\end{gathered}
$$

where $z:=\operatorname{col}\left(z_{1}, \ldots, z_{n}\right), z_{i}:=\operatorname{col}\left(z_{i 1}^{a}, z_{i}^{b}, z_{i 2}^{a}\right), z_{i 1}^{a}:=\operatorname{col}\left(z_{i 11}, z_{i 12}, \cdots z_{i 1\left(r_{i 1}-1\right)}, \cdots, z_{i m_{i} 1}, z_{i m_{i} 2}, \cdots, z_{i m_{i}\left(r_{i m_{i}}-1\right)}\right) \in$ $R^{r_{i}-m_{i}}, z_{i 2}^{a}:=\left(z_{i 1 r_{i 1}}, z_{i 2 r_{i 2}}, \cdots, z_{i m_{i} r_{i m_{i}}}\right) \in R^{m_{i}}, \Psi_{i 1}^{a}(t, z), \Psi_{i}^{b}(t, z), \Psi_{i 2}^{a}(t, z)$ are the interconnections with appropriate dimensions, $\Xi_{i 1}^{a}\left(t, z_{i}\right), \Xi_{i}^{b}\left(t, z_{i}\right), \Xi_{i 2}^{a}\left(t, z_{i}\right)$ are the uncertainties with appropriate dimensions. Taking into account the special structure of $\left(\bar{A}_{i}, \bar{B}_{i}\right)$ in (10), form (11) can be obtained by matrix factorization to $\left(\bar{A}_{i}, \bar{B}_{i}\right)$, while $\left(A_{i}, B_{i}\right)$ also has a similar structure to $\left(\bar{A}_{i}, \bar{B}_{i}\right)$.

In particular, $A_{i}=\operatorname{diag}\left\{A_{i 1}, \cdots, A_{i l}, \cdots, A_{i m_{i}}\right\} \in R^{\left(r_{i}-m_{i}\right) \times\left(r_{i}-m_{i}\right)}, B_{i}=\operatorname{diag}\left\{B_{i 1}, \cdots, B_{i l}, \cdots, B_{i m_{i}}\right\} \in R^{\left(r_{i}-m_{i}\right) \times m_{i}}$ with

$$
A_{i l}=\left[\begin{array}{cccccc}
0 & 1 & 0 & \vdots & 0 & 0 \\
0 & 0 & 1 & \vdots & 0 & 0 \\
\vdots & \vdots & \vdots & \ddots & \vdots & \vdots \\
0 & 0 & 0 & \cdots & 0 & 1 \\
0 & 0 & 0 & \cdots & 0 & 0
\end{array}\right]_{\left(r_{l}-1\right) \times\left(r_{l}-1\right)} \quad, B_{i l}=\left[\begin{array}{c}
0 \\
0 \\
\vdots \\
0 \\
1
\end{array}\right]_{\left(r_{l}-1\right) \times 1}
$$


Remark 2: Under the diffeomorphism $T_{i}$ defined in (6) and $T_{i}(0)=0$, the uncertainties and interconnections of the interconnected system (11) have a similar structure to those of the interconnected system (1). For example, $\Psi_{i 1}^{a}(t, z), \Psi_{i}^{b}(t, z)$ and $\Psi_{i 2}^{a}(t, z)$ are determined by $\left.\left[\frac{\partial T_{i}}{\partial x_{i}}\right]\right|_{x_{i}=T_{i}^{-1}\left(z_{i}\right)} \sum_{j=1}^{n} H_{i j}\left(t, T_{j}^{-1}\left(z_{j}\right)\right)$.

Remark 3: It is clear to see that the system (11) adopts a regular form as $\nu_{i}$ is the system input, which has been transformed from the nonlinear interconnected system (1) by local coordinate transformation and feedback linearization. It should be noted that form (10) is directly studied in [23] with the non-square matrix $\bar{B}_{i} \in R^{r_{i} \times m_{i}}$ as the input distribution matrix. Based on a high gain control approach, a decentralised state feedback control scheme is proposed for this form, which can only deal with weak unmatched uncertainties and matched interconnections [23]. Furthermore, the decentralised sliding mode control cannot be directly designed in the light of form (10). A new form (11) is developed in this study with nonsingular unit matrix as the new input distribution matrix, which not only facilitates the design of the sliding mode control, but also can deal with stronger unmatched uncertainties and interconnections. Note that form (11) can be obtained by a simple matrix factorization technique from (10).

The objective of this paper is to design a composite sliding surface under a set of conditions such that the reduced sliding mode is uniformly asymptotically stable. Then, for system (11), a decentralised sliding mode control

$$
\nu_{i}=\nu_{i}\left(t, z_{i}\right)
$$

will be designed such that the controlled interconnected system states can be driven to the pre-designed composite sliding surface in finite time and a sliding motion maintained thereafter.

Remark 4: For system (1), a constructive method to obtain the required regular form is not available [27]. In comparison with [27], the proposed controller design in this paper has more practical significance and application value in this regard. It will be shown that in the developed regular form (11), the method proposed in this paper can accommodate the case of unmatched uncertainties and interconnections.

\section{BASIC ASSUMPTIONS AND STABILITY ANALYSIS OF THE SLIDING MODE}

In this section, a composite sliding surface will be designed. Then the stability of the corresponding sliding motion will be analysed. The following Assumption will be imposed on system (11).

Assumption 1: There exist known nonnegative continuous functions $\mu_{i}(\cdot), \rho_{i}(\cdot), \alpha_{i 1}(\cdot), \alpha_{i 2}(\cdot), \beta_{i 1}(\cdot), \beta_{i 2}(\cdot), \gamma_{i 1}(\cdot), \gamma_{i 2}(\cdot)$, $\alpha_{i j 3}(\cdot), \alpha_{i j 4}(\cdot), \alpha_{i j 5}(\cdot), \beta_{i j 3}(\cdot), \beta_{i j 4}(\cdot), \beta_{i j 5}(\cdot), \gamma_{i j 3}(\cdot), \gamma_{i j 4}(\cdot), \gamma_{i j 5}(\cdot)$, such that

$$
\begin{gathered}
\left\|\Xi_{i 2}^{a}\left(t, z_{i}\right)\right\| \leqslant \mu_{i}\left(z_{i}\right) \\
\left\|\Xi_{i 1}^{a}\left(t, z_{i}\right)\right\| \leqslant \rho_{i}\left(z_{i}\right)=\alpha_{i 1}\left(z_{i}\right)\left\|z_{i 1}^{a}\right\|+\beta_{i 1}\left(z_{i}\right)\left\|z_{i 2}^{a}\right\|+\gamma_{i 1}\left(z_{i}\right)\left\|z_{i}^{b}\right\| \\
\left\|\Xi_{i}^{b}\left(t, z_{i}\right)\right\| \leqslant \alpha_{i 2}\left(z_{i}\right)\left\|z_{i 1}^{a}\right\|+\beta_{i 2}\left(z_{i}\right)\left\|z_{i 2}^{a}\right\|+\gamma_{i 2}\left(z_{i}\right)\left\|z_{i}^{b}\right\| \\
\left\|\Psi_{i 1}^{a}(t, z)\right\| \leqslant \sum_{j=1}^{n}\left(\alpha_{i j 3}\left(z_{j}\right)\left\|z_{j 1}^{a}\right\|+\beta_{i j 3}\left(z_{j}\right)\left\|z_{j 2}^{a}\right\|+\gamma_{i j 3}\left(z_{j}\right)\left\|z_{j}^{b}\right\|\right) \\
\left\|\Psi_{i 2}^{a}(t, z)\right\| \leqslant \sum_{j=1}^{n}\left(\alpha_{i j 4}\left(z_{j}\right)\left\|z_{j 1}^{a}\right\|+\beta_{i j 4}\left(z_{j}\right)\left\|z_{j 2}^{a}\right\|+\gamma_{i j 4}\left(z_{j}\right)\left\|z_{j}^{b}\right\|\right) \\
\left\|\Psi_{i}^{b}(t, z)\right\| \leqslant \sum_{j=1}^{n}\left(\alpha_{i j 5}\left(z_{j}\right)\left\|z_{j 1}^{a}\right\|+\beta_{i j 5}\left(z_{j}\right)\left\|z_{j 2}^{a}\right\|+\gamma_{i j 5}\left(z_{j}\right)\left\|z_{j}^{b}\right\|\right)
\end{gathered}
$$

Remark 5: Assumption 1 is made on the uncertainties and interconnections in the nonlinear interconnected system (11). Meanwhile, Remark 2 shows that the bounds on the uncertainties and interconnections in the interconnected system (1) have a 
similar structure to those in the interconnected system (11). The terms $\Xi_{i 1}^{a}\left(t, z_{i}\right)$ and $\Xi_{i}^{b}\left(t, z_{i}\right)$ represent unmatched uncertainties, while $\Psi_{i 1}^{a}(t, z)$ and $\Psi_{i}^{b}(t, z)$ represent unmatched interconnections. It should be noted that only matched uncertainties and interconnections are discussed in [27] and [23] respectively, while unmatched uncertainties and interconnections are also considered in this paper. Furthermore, the bounds on the uncertainties and interconnections are nonlinear and have more general forms.

It is clear that $\left(A_{i l}, B_{i l}\right)$ in (12) have the Brunovsky standard form [30], and according to the controllability criterion, $\left(A_{i}, B_{i}\right)$ are controllable. It follows that there exist $K_{i} \in R^{m_{i} \times\left(r_{i}-m_{i}\right)}$ such that the matrices $\left(A_{i}-B_{i} K_{i}\right)$ are stable. Therefore, there exist positive definite symmetric matrices $Q_{i}, P_{i}$ such that

$$
\left(A_{i}-B_{i} K_{i}\right)^{T} P_{i}+P_{i}\left(A_{i}-B_{i} K_{i}\right)+Q_{i} \leqslant 0, i=1,2, \ldots, n
$$

Remark 6: It should be noted that matrix inequalities (16) can be transformed into the following standard linear matrix inequalities (LMI) (see e.g. [31], [32]). Given $Q_{i}>0$, find matrices $P_{i}$ and $K_{i}$ such that:

$$
\left[\begin{array}{cc}
A_{i}^{T} P_{i}+P_{i} A_{i}-F_{i}^{T}-F_{i} & I_{i} \\
I_{i} & -Q_{i}^{-1}
\end{array}\right] \leqslant 0
$$

where $F_{i}=P_{i} B_{i} K_{i}$ and $I_{i}$ are unit matrices for $i=1,2, \ldots, n$.

Choose the composite sliding surface

$$
\sigma(z)=\operatorname{col}\left(\sigma_{1}\left(z_{1}\right), \cdots, \sigma_{n}\left(z_{n}\right)\right)=0
$$

where $\sigma_{i}\left(z_{i}\right)=z_{i 2}^{a}+K_{i} z_{i 1}^{a}$ and $K_{i}$ satisfies (16).

During sliding motion $\sigma_{i}\left(z_{i}\right)=0$ which implies

$$
z_{i 2}^{a}=-K_{i} z_{i 1}^{a}
$$

Based on the designed sliding surface (18) and (19), the corresponding sliding motion can be determined using (11). The sliding mode dynamics are described by:

$$
\begin{aligned}
& \dot{z}_{i 1}^{a}=\left(A_{i}-B_{i} K_{i}\right) z_{i 1}^{a}+\mathrm{T}_{i}^{a s}(\cdot)+\Upsilon_{i}^{a s}(\cdot) \\
& \dot{z}_{i}^{b}=\Omega_{i}(\cdot)+\mathrm{T}_{i}^{b s}(\cdot)+\Upsilon_{i}^{b s}(\cdot)
\end{aligned}
$$

where $T_{i}^{a s}\left(t, z_{11}^{a}, z_{1}^{b}, \cdots z_{n 1}^{a}, z_{n}^{b}\right)=\left.\Psi_{i 1}^{a}(t, z)\right|_{z_{i 2}^{a}=-K_{i} z_{i 1}^{a}}, \Upsilon_{i}^{a s}\left(t, z_{i 1}^{a}, z_{i}^{b}\right)=\left.\Xi_{i 1}^{a}\left(t, z_{i}\right)\right|_{z_{i 2}^{a}=-K_{i} z_{i 1}^{a}}, \mathrm{~T}_{i}^{b s}\left(t, z_{11}^{a}, z_{1}^{b}, \cdots z_{n 1}^{a}, z_{n}^{b}\right)=$ $\left.\Psi_{i}^{b}(t, z)\right|_{z_{i 2}^{a}=-K_{i} z_{i 1}^{a}}, \Upsilon_{i}^{b s}\left(t, z_{i 1}^{a}, z_{i}^{b}\right)=\left.\Xi_{i}^{b}\left(t, z_{i}\right)\right|_{z_{i 2}^{a}=-K_{i} z_{i 1}^{a}}, \Omega_{i}\left(z_{i 1}^{a}, z_{i}^{b}\right)=\left.\omega_{i}\left(z_{i 1}^{a}, z_{i 2}^{a}, z_{i}^{b}\right)\right|_{z_{i 2}^{a}=-K_{i} z_{i 1}^{a}}$.

Assumption 2: There exist $C^{1}$ functions $V_{i}^{b}\left(t, z_{i 1}^{a}, z_{i}^{b}\right): R \times R^{r_{i}-m_{i}} \times R^{n_{i}-r_{i}} \mapsto R^{+}$such that

$$
\begin{gathered}
c_{i 1}\left\|z_{i 1}^{a}\right\|^{2}+c_{i 2}\left\|z_{i}^{b}\right\|^{2} \leqslant V_{i}^{b}\left(t, z_{i 1}^{a}, z_{i}^{b}\right) \leqslant c_{i 3}\left\|z_{i 1}^{a}\right\|^{2}+c_{i 4}\left\|z_{i}^{b}\right\|^{2} \\
\frac{\partial V_{i}^{b}}{\partial t}+\left(\frac{\partial V_{i}^{b}}{\partial z_{i 1}^{a}}\right)^{T}\left(A_{i}-B_{i} K_{i}\right) z_{i 1}^{a}+\left(\frac{\partial V_{i}^{b}}{\partial z_{i}^{b}}\right)^{T} \Omega_{i}\left(z_{i 1}^{a}, z_{i}^{b}\right) \leqslant-c_{i 5}\left\|z_{i 1}^{a}\right\|^{2}-c_{i 6}\left\|z_{i}^{b}\right\|^{2} \\
\left\|\frac{\partial V_{i}^{b}}{\partial\left(z_{i 1}^{a}, z_{i}^{b}\right)}\right\| \leqslant c_{i 7}\left\|z_{i 1}^{a}\right\|+c_{i 8}\left\|z_{i}^{b}\right\|
\end{gathered}
$$

where $c_{i 1}, \cdots, c_{i 8}$ are positive constants, $\frac{\partial V_{i}^{b}}{\partial\left(z_{i 1}^{a}, z_{i}^{b}\right)}=\left[\left(\frac{\partial V_{i}^{b}}{\partial z_{i 1}^{a}}\right)^{T}\left(\frac{\partial V_{i}^{b}}{\partial z_{i}^{b}}\right)^{T}\right], K_{i}$ satisfies $(16)$ and $\Omega_{i}\left(z_{i 1}^{a}, z_{i}^{b}\right)$ is given in (20).

Remark 7: Assumption 2 implies that the nominal isolated subsystems of (20) are asymptotically stable. Such conditions are derived from the converse Lyapunov uniform asymptotic stability theorem (see e.g. [33]).

From Assumption $1, \mathrm{~T}_{i}^{a s}(\cdot), \Upsilon_{i}^{a s}(\cdot), \mathrm{T}_{i}^{b s}(\cdot)$ and $\Upsilon_{i}^{b s}(\cdot)$ in (20) satisfy

$$
\left\|\mathrm{T}_{i}^{a s}\left(t, z_{11}^{a}, z_{1}^{b}, \cdots z_{n 1}^{a}, z_{n}^{b}\right)\right\| \leqslant \sum_{j=1}^{n}\left(\varepsilon_{i j 1}\left(z_{j 1}^{a}, z_{j}^{b}\right)\left\|z_{j 1}^{a}\right\|+\varepsilon_{i j 2}\left(z_{j 1}^{a}, z_{j}^{b}\right)\left\|z_{j}^{b}\right\|\right)
$$




$$
\begin{gathered}
\left\|\mathrm{T}_{i}^{b s}\left(t, z_{11}^{a}, z_{1}^{b}, \cdots z_{n 1}^{a}, z_{n}^{b}\right)\right\| \leqslant \sum_{j=1}^{n}\left(\varepsilon_{i j 5}\left(z_{j 1}^{a}, z_{j}^{b}\right)\left\|z_{j 1}^{a}\right\|+\varepsilon_{i j 6}\left(z_{j 1}^{a}, z_{j}^{b}\right)\left\|z_{j}^{b}\right\|\right) \\
\left\|\Upsilon_{i}^{a s}\left(t, z_{i 1}^{a}, z_{i}^{b}\right)\right\| \leqslant \varepsilon_{i 3}\left(z_{i 1}^{a}, z_{i}^{b}\right)\left\|z_{i 1}^{a}\right\|+\varepsilon_{i 4}\left(z_{i 1}^{a}, z_{i}^{b}\right)\left\|z_{i}^{b}\right\| \\
\left\|\Upsilon_{i}^{b s}\left(t, z_{i 1}^{a}, z_{i}^{b}\right)\right\| \leqslant \varepsilon_{i 7}\left(z_{i 1}^{a}, z_{i}^{b}\right)\left\|z_{i 1}^{a}\right\|+\varepsilon_{i 8}\left(z_{i 1}^{a}, z_{i}^{b}\right)\left\|z_{i}^{b}\right\|
\end{gathered}
$$

where

$$
\begin{gathered}
\varepsilon_{i j 1}\left(z_{j 1}^{a}, z_{j}^{b}\right)=\left.\left\{\alpha_{i j 3}\left(z_{j}\right)+\beta_{i j 3}\left(z_{j}\right)\left\|K_{j}\right\|\right\}\right|_{z_{j 2}^{a}=-K_{j} z_{j 1}^{a}} \\
\varepsilon_{i j 2}\left(z_{j 1}^{a}, z_{j}^{b}\right)=\left.\gamma_{i j 3}\left(z_{j}\right)\right|_{z_{j 2}^{a}=-K_{j} z_{j 1}^{a}} \\
\varepsilon_{i 3}\left(z_{i 1}^{a}, z_{i}^{b}\right)=\left.\left\{\alpha_{i 1}\left(z_{j}\right)+\beta_{i 1}\left(z_{j}\right)\left\|K_{i}\right\|\right\}\right|_{z_{i 2}^{a}=-K_{i} z_{i 1}^{a}} \\
\varepsilon_{i 4}\left(z_{i 1}^{a}, z_{i}^{b}\right)=\left.\gamma_{i 1}\left(z_{i}\right)\right|_{z_{i 2}^{a}=-K_{i} z_{i 1}^{a}} \\
\varepsilon_{i j 5}\left(z_{j 1}^{a}, z_{j}^{b}\right)=\left.\left\{\alpha_{i j 5}\left(z_{j}\right)+\beta_{i j 5}\left(z_{j}\right)\left\|K_{j}\right\|\right\}\right|_{z_{j 2}^{a}=-K_{j} z_{j 1}^{a}} \\
\varepsilon_{i j 6}\left(z_{j 1}^{a}, z_{j}^{b}\right)=\left.\gamma_{i j 5}\left(z_{j}\right)\right|_{z_{j 2}^{a}=-K_{j} z_{j 1}^{a}} \\
\varepsilon_{i 7}\left(z_{i 1}^{a}, z_{i}^{b}\right)=\left.\left\{\alpha_{i 2}\left(z_{i}\right)+\beta_{i 2}\left(z_{i}\right)\left\|K_{i}\right\|\right\}\right|_{z_{i 2}^{a}=-K_{i} z_{i 1}^{a}} \\
\varepsilon_{i 8}\left(z_{i 1}^{a}, z_{i}^{b}\right)=\left.\gamma_{i 2}\left(z_{i}\right)\right|_{z_{i 2}^{a}=-K_{i} z_{i 1}^{a}}
\end{gathered}
$$

Theorem 1: Under Assumptions 1-2, the sliding mode dynamics (20) of system (11) are asymptotically stable if $M^{T}+M>0$ where the matrix $M=\left[m_{i j}\right]_{2 n \times 2 n}$ is defined by

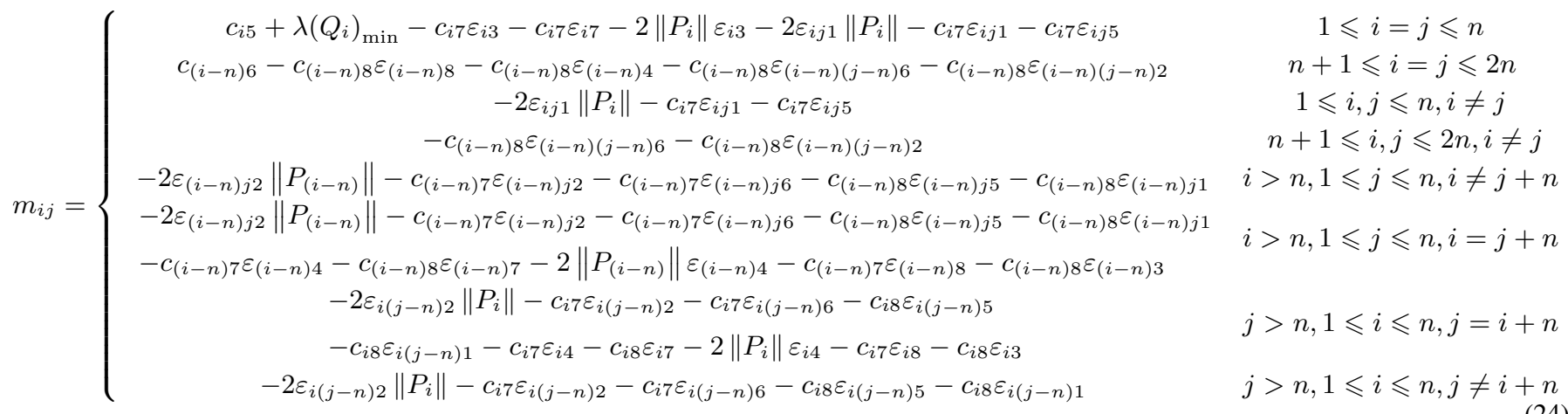

where $\lambda(\cdot)_{\min }$ represents the minimum eigenvalue of $(\cdot)$.

Proof: For system (20), consider the Lyapunov function candidate

$$
V(t, z)=\sum_{i=1}^{n} V_{i}^{b}\left(t, z_{i 1}^{a}, z_{i}^{b}\right)+\sum_{i=1}^{n}\left(z_{i 1}^{a}\right)^{T} P_{i} z_{i 1}^{a}
$$

where $V_{i}^{b}\left(t, z_{i 1}^{a}, z_{i}^{b}\right)$ is given by Assumption 2, and $P_{i}$ satisfies (16). 
Then, the time derivative of $V(t, z)$ along the trajectories of system (20) is given by:

$$
\begin{aligned}
\dot{V} & =\sum_{i=1}^{n}\left\{\frac{\partial V_{i}^{b}}{\partial t}+\frac{\partial V_{i}^{b}}{\partial\left(z_{i 1}^{a}, z_{i}^{b}\right)}\left[\begin{array}{c}
\dot{z}_{i 1}^{a} \\
\dot{z}_{i}^{b}
\end{array}\right]\right\}+\sum_{i=1}^{n}\left\{-\left(z_{i 1}^{a}\right)^{T} Q_{i} z_{i 1}^{a}+2\left(z_{i 1}^{a}\right)^{T} P_{i}\left(\mathrm{~T}_{i}^{a s}+\Upsilon_{i}^{a s}\right)\right\} \\
& =\sum_{i=1}^{n}\left\{\frac{\partial V_{i}^{b}}{\partial t}+\left[\left(\frac{\partial V_{i}^{b}}{\partial z_{i 1}^{a}}\right)^{T}\left(\frac{\partial V_{i}^{b}}{\partial z_{i}^{b}}\right)^{T}\right]\left[\begin{array}{c}
\left(A_{i}-B_{i} K_{i}\right) z_{i 1}^{a}+\mathrm{T}_{i}^{a s}+\Upsilon_{i}^{a s} \\
\Omega_{i}\left(z_{i 1}^{a}, z_{i}^{b}\right)+\mathrm{T}_{i}^{b s}+\Upsilon_{i}^{b s}
\end{array}\right]\right\}+\sum_{i=1}^{n}\left\{-\left(z_{i 1}^{a}\right)^{T} Q_{i} z_{i 1}^{a}+2\left(z_{i 1}^{a}\right)^{T} P_{i}\left(\mathrm{~T}_{i}^{a s}+\Upsilon_{i}^{a s}\right)\right\}
\end{aligned}
$$

where $Q_{i}=-\left(A_{i}-B_{i} K_{i}\right)^{T} P_{i}-P_{i}\left(A_{i}-B_{i} K_{i}\right)$ has been used for convenience.

Hence, (26) can be expressed as:

$$
\begin{aligned}
\dot{V} & \leqslant \sum_{i=1}^{n}\left\{\frac{\partial V_{i}^{b}}{\partial t}+\left(\frac{\partial V_{i}^{b}}{\partial z_{i 1}^{a}}\right)^{T}\left(A_{i}-B_{i} K_{i}\right) z_{i 1}^{a}+\left(\frac{\partial V_{i}^{b}}{\partial z_{i}^{b}}\right)^{T} \Omega_{i}\left(z_{i 1}^{a}, z_{i}^{b}\right)+\left\|\frac{\partial V_{i}^{b}}{\partial\left(z_{i 1}^{a}, z_{i}^{b}\right)}\right\|\left\|\left[\begin{array}{c}
\mathrm{T}_{i}^{a s}+\Upsilon_{i}^{a s} \\
\mathrm{~T}_{i}^{b s}+\Upsilon_{i}^{b s}
\end{array}\right]\right\|\right\} \\
& +\sum_{i=1}^{n}\left\{-\left(z_{i 1}^{a}\right)^{T} Q_{i} z_{i 1}^{a}+2\left(z_{i 1}^{a}\right)^{T} P_{i}\left(\mathrm{~T}_{i}^{a s}+\Upsilon_{i}^{a s}\right)\right\}
\end{aligned}
$$

Then, from Assumptions 2 and (27), the following inequalities can be obtained:

$$
\begin{aligned}
\dot{V} & \leqslant \sum_{i=1}^{n}\left\{-c_{i 5}\left\|z_{i 1}^{a}\right\|^{2}-c_{i 6}\left\|z_{i}^{b}\right\|^{2}+\left(c_{i 7}\left\|z_{i 1}^{a}\right\|+c_{i 8}\left\|z_{i}^{b}\right\|\right)\left(\left\|\mathrm{T}_{i}^{a s}\right\|+\left\|\Upsilon_{i}^{a s}\right\|\right)\right. \\
& \left.+\left(c_{i 7}\left\|z_{i 1}^{a}\right\|+c_{i 8}\left\|z_{i}^{b}\right\|\right)\left(\left\|\mathrm{T}_{i}^{b s}\right\|+\left\|\Upsilon_{i}^{b s}\right\|\right)\right\} \\
& +\sum_{i=1}^{n}\left\{-\lambda\left(Q_{i}\right)_{\min }\left\|z_{i 1}^{a}\right\|^{2}+2\left\|z_{i 1}^{a}\right\|\left\|P_{i}\right\|\left\|\mathrm{T}_{i}^{a s}\right\|+2\left\|z_{i 1}^{a}\right\|\left\|P_{i}\right\|\left\|\Upsilon_{i}^{a s}\right\|\right\}
\end{aligned}
$$

From the inequalities (22) and (23), it follows that

$$
\begin{aligned}
\dot{V} & \leqslant \sum_{i=1}^{n}\left\{\left\{-c_{i 5}-\lambda\left(Q_{i}\right)_{\min }+c_{i 7} \varepsilon_{i 3}+c_{i 7} \varepsilon_{i 7}+2\left\|P_{i}\right\| \varepsilon_{i 3}\right\}\left\|z_{i 1}^{a}\right\|^{2}+\left\{-c_{i 6}+c_{i 8} \varepsilon_{i 8}+c_{i 8} \varepsilon_{i 4}\right\}\left\|z_{i}^{b}\right\|^{2}\right. \\
& \left.+\left\{c_{i 7} \varepsilon_{i 4}+c_{i 8} \varepsilon_{i 7}+2\left\|P_{i}\right\| \varepsilon_{i 4}+c_{i 7} \varepsilon_{i 8}+c_{i 8} \varepsilon_{i 3}\right\}\left\|z_{i 1}^{a}\right\|\left\|z_{i}^{b}\right\|\right\} \\
& +\sum_{i=1}^{n} \sum_{j=1}^{n}\left\{2 \varepsilon_{i j 1}\left\|P_{i}\right\|+c_{i 7} \varepsilon_{i j 1}+c_{i 7} \varepsilon_{i j 5}\right\}\left\|z_{i 1}^{a}\right\|\left\|z_{j 1}^{a}\right\|+\sum_{i=1}^{n} \sum_{j=1}^{n}\left\{2 \varepsilon_{i j 2}\left\|P_{i}\right\|+c_{i 7} \varepsilon_{i j 2}+c_{i 7} \varepsilon_{i j 6}\right\}\left\|z_{i 1}^{a}\right\|\left\|z_{j}^{b}\right\| \\
& +\sum_{i=1}^{n} \sum_{j=1}^{n}\left\{c_{i 8} \varepsilon_{i j 6}+c_{i 8} \varepsilon_{i j 2}\right\}\left\|z_{i}^{b}\right\|\left\|z_{j}^{b}\right\|+\sum_{i=1}^{n} \sum_{j=1}^{n}\left\{c_{i 8} \varepsilon_{i j 5}+c_{i 8} \varepsilon_{i j 1}\right\}\left\|z_{i}^{b}\right\|\left\|z_{j 1}^{a}\right\| \\
& =-\frac{1}{2} Y\left(M+M^{T}\right) Y^{T}
\end{aligned}
$$

where $Y=\left[\left\|z_{11}^{a}\right\|,\left\|z_{21}^{a}\right\|, \cdots,\left\|z_{n 1}^{a}\right\|,\left\|z_{1}^{b}\right\|,\left\|z_{2}^{b}\right\|, \cdots,\left\|z_{n}^{b}\right\|\right]$.

Hence, due to $M^{T}+M>0$, Theorem 1 follows.

Remark 8: It should be noted noted that for $M \in R^{2 n \times 2 n}$, Theorem 1 has presented a set of sufficient conditions under which the sliding mode (20) of the nonlinear interconnected system (11) is asymptotically stable even in the presence of unmatched uncertainties and interconnections.

\section{DECEnTRALISED SLIDING MODE CONTROL DESIGN}

This section aims to design a decentralised sliding mode control such that system (11) can be driven to the sliding surface (18) in finite time.

Lemma 1: For the nonlinear interconnected system (11) with the sliding surface given in (18), if

$$
\sum_{i=1}^{n} \sigma_{i}^{T}\left(z_{i}\right) \dot{\sigma}_{i}\left(z_{i}\right) \leqslant-\eta \sum_{i=1}^{n}\left\|\sigma_{i}\left(z_{i}\right)\right\|
$$

where $\eta$ is a small positive constant, then the system (11) can reach the sliding surface (18) in finite time and maintain a sliding motion thereafter. 
Proof: For the nonlinear interconnected system, composite switching function $\sigma(z)=\operatorname{col}\left(\sigma_{1}\left(z_{1}\right), \cdots, \sigma_{n}\left(z_{n}\right)\right)$, the following equation holds:

$$
\sigma^{T}(z) \dot{\sigma}(z)=\sum_{i=1}^{n} \sigma_{i}^{T}\left(z_{i}\right) \dot{\sigma}_{i}\left(z_{i}\right)
$$

Furthermore,

$$
\sum_{i=1}^{n}\left\|\sigma_{i}\left(z_{i}\right)\right\|=\left\|\sigma_{1}\left(z_{1}\right)\right\|+\cdots+\left\|\sigma_{n}\left(z_{n}\right)\right\| \geqslant\|\sigma(z)\|=\sqrt{\left\|\sigma_{1}\left(z_{1}\right)\right\|^{2}+\cdots+\left\|\sigma_{n}\left(z_{n}\right)\right\|^{2}}
$$

From (31) and (32),

$$
\sigma^{T}(z) \dot{\sigma}(z)=\sum_{i=1}^{n} \sigma_{i}^{T}\left(z_{i}\right) \dot{\sigma}_{i}\left(z_{i}\right) \leqslant-\eta \sum_{i=1}^{n}\left\|\sigma_{i}\left(z_{i}\right)\right\| \leqslant-\eta\|\sigma(z)\|
$$

Hence, Lemma 1 follows since a so-called $\eta$ reachability condition is satisfied [34].

Remark 9: In the existing research concerning nonlinear interconnected systems, the corresponding reachability condition is given by: (see, [35])

$$
\sum_{i=1}^{n} \frac{\sigma_{i}^{T}\left(z_{i}\right) \dot{\sigma}_{i}\left(z_{i}\right)}{\left\|\sigma_{i}\left(z_{i}\right)\right\|}<0
$$

Condition (34) cannot guarantee that the control drives the system states to the sliding surface in finite time. However, the reachability condition proposed in (30) in this paper can guarantee that the system states can be driven to the sliding surface in finite time and also maintain a sliding motion thereafter.

For the interconnected system (11), the following control law is proposed

$$
\begin{aligned}
\nu_{i} & =-K_{i} A_{i} z_{i 1}^{a}-K_{i} B_{i} z_{i 2}^{a}-\mu_{i}\left(z_{i}^{a}\right) \operatorname{sgn}\left(\sigma_{i}\left(z_{i}\right)\right)-\rho_{i}\left(z_{i}^{a}, z_{i 2}^{a}, z_{i}^{b}\right)\left\|K_{i}\right\| \operatorname{sgn}\left(\sigma_{i}\left(z_{i}\right)\right) \\
& -\Phi_{i} \operatorname{sgn}\left(\sigma_{i}\left(z_{i}\right)\right)-\eta \operatorname{sgn}\left(\sigma_{i}\left(z_{i}\right)\right)
\end{aligned}
$$

where $K_{i}$ is given in (16) and

$$
\begin{aligned}
\Phi_{i} & =\sum_{j=1}^{n}\left\{\left(\alpha_{j i 4}\left(z_{i}\right)+\left\|K_{j}\right\| \alpha_{j i 3}\left(z_{i}\right)\right)\left\|z_{i 1}^{a}\right\|+\left(\beta_{j i 4}\left(z_{i}\right)+\left\|K_{j}\right\| \beta_{j i 3}\left(z_{i}\right)\right)\left\|z_{i 2}^{a}\right\|\right. \\
& \left.+\left(\gamma_{j i 4}\left(z_{i}\right)+\left\|K_{j}\right\| \gamma_{j i 3}\left(z_{i}\right)\right)\left\|z_{i}^{b}\right\|\right\}
\end{aligned}
$$

Theorem 2: Under Assumptions 1-2, the control (35) is able to drive the system (11) to the sliding surface (18) in finite time and maintain a sliding motion on it thereafter.

Proof: From (11) and (18), for $i=1,2, \cdots n$,

$$
\begin{aligned}
\dot{\sigma}_{i}\left(z_{i}\right) & =\dot{z}_{i 2}^{a}+K_{i} \dot{z}_{i 1}^{a} \\
& =\nu_{i}+\Psi_{i 2}^{a}(t, z)+\Xi_{i 2}^{a}\left(t, z_{i}\right)+K_{i}\left(A_{i} z_{i 1}^{a}+B_{i} z_{i 2}^{a}+\Psi_{i 1}^{a}(t, z)+\Xi_{i 1}^{a}\left(t, z_{i}\right)\right)
\end{aligned}
$$

Substituting (35) into (37),

$$
\begin{aligned}
\sum_{i=1}^{n} \sigma_{i}^{T}\left(z_{i}\right) \dot{\sigma}_{i}\left(z_{i}\right) & =\sum_{i=1}^{n} \sigma_{i}^{T}\left(z_{i}\right)\left\{-\mu_{i}\left(z_{i}\right) \operatorname{sgn}\left(\sigma_{i}\left(z_{i}\right)\right)-\rho_{i}\left(z_{i}\right)\left\|K_{i}\right\| \operatorname{sgn}\left(\sigma_{i}\left(z_{i}\right)\right)\right. \\
& -\Phi_{i} \operatorname{sgn}\left(\sigma_{i}\left(z_{i}\right)\right)-\eta \operatorname{sgn}\left(\sigma_{i}\left(z_{i}\right)\right)+\Psi_{i 2}^{a}(t, z)+\Xi_{i 2}^{a}\left(t, z_{i}\right) \\
& \left.+K_{i} \Psi_{i 1}^{a}(t, z)+K_{i} \Xi_{i 1}^{a}\left(t, z_{i}\right)\right\}
\end{aligned}
$$


From Assumptions 1 and the definition of $\Phi_{i}$ in (36), the following inequality can be obtained:

$$
\begin{aligned}
\sum_{i=1}^{n} \sigma_{i}^{T}\left(z_{i}\right) \dot{\sigma}_{i}\left(z_{i}\right) & \leqslant \sum_{i=1}^{n}\left\{-\mu_{i}\left(z_{i}\right)\left\|\sigma_{i}\left(z_{i}\right)\right\|-\rho_{i}\left(z_{i}\right)\left\|K_{i}\right\|\left\|\sigma_{i}\left(z_{i}\right)\right\|+\mu_{i}\left(z_{i}\right)\left\|\sigma_{i}\left(z_{i}\right)\right\|\right. \\
& \left.+\rho_{i}\left(z_{i}\right)\left\|K_{i}\right\|\left\|\sigma_{i}\left(z_{i}\right)\right\|-\eta\left\|\sigma_{i}\left(z_{i}\right)\right\|\right\}+\sum_{i=1}^{n}\left\{-\Phi_{i}\left\|\sigma_{i}\left(z_{i}\right)\right\|\right. \\
& +\sum_{j=1}^{n}\left\{\left(\alpha_{i j 4}\left(z_{j}\right)+\left\|K_{i}\right\| \alpha_{i j 3}\left(z_{j}\right)\right)\left\|z_{j 1}^{a}\right\|+\left(\beta_{i j 4}\left(z_{j}\right)+\left\|K_{i}\right\| \beta_{i j 3}\left(z_{j}\right)\right)\left\|z_{j 2}^{a}\right\|\right. \\
& \left.\left.+\left(\gamma_{i j 4}\left(z_{j}\right)+\left\|K_{i}\right\| \gamma_{i j 3}\left(z_{j}\right)\right)\left\|z_{j}^{b}\right\|\right\}\left\|\sigma_{i}\left(z_{i}\right)\right\|\right\} \\
& =-\eta \sum_{i=1}^{n}\left\|\sigma_{i}\left(z_{i}\right)\right\|
\end{aligned}
$$

where $\sum_{i=1}^{n} \sum_{j=1}^{n} \Phi_{i}=\sum_{i=1}^{n} \sum_{j=1}^{n} \Phi_{j}$ has been used to generate the result.

Hence, from Lemma 1, Theorem 2 follows.

Remark 10: Theorems 1 and 2 together guarantee the uniform asymptotic stability of the closed-loop system formed by applying the control (35) to the system (11), irrespective of the uncertainties and the interconnections within the subsystems. Meanwhile, it should be noted that from the relationship between systems (1) and (11), it is straightforward to rewrite the control (9) and (35) in terms of the $x$ coordinates to stabilize system (1) with $x_{i}=T_{i}^{-1}\left(z_{i}\right)$ and $T_{i}$ defined in (6). It should be noted that the results of this paper are local. A method to estimate the stability region has been studied in [33], [36], [37].

Remark 11: There are few results which apply sliding mode control methods to the nonlinear interconnected system described by (1). Since the nominal isolated system in (1) is completely nonlinear and experiences unmatched uncertainties and interconnections, the control design is particularly challenging. Based on local coordinate transformation and feedback linearization, the considered interconnected system (1) is transformed to a new regular form (11). IA composite sliding surface (18) is then proposed and a set of sufficient conditions is developed to guarantee that the corresponding sliding mode dynamics (20) is asymptotically stable even in the presence of unmatched uncertainties and interconnections. A new reachability condition (30) is introduced and used to define a decentralised sliding mode control law (35) that can drive the system (11) to the sliding surface (18) in finite time and maintain a sliding motion on it thereafter.

\section{Simulation example}

This section aims to demonstrate the validity of the proposed method by a simulation example using the MATLAB software. Consider the nonlinear interconnected system formed of the two subsystems described by:

$$
\begin{gathered}
\dot{x}_{1}=\left[\begin{array}{c}
-\sin x_{12} \\
x_{11} \\
x_{12}^{2}+x_{13}^{2} \\
x_{13} \\
2 x_{11} x_{12}-4 x_{15}
\end{array}\right]+\left[\begin{array}{cc}
1 & 0 \\
0 & 0 \\
0 & 2+\sin x_{15} \\
0 & 0 \\
0 & 0
\end{array}\right]\left(u_{1}+\phi_{1}\left(t, x_{1}\right)\right)+\left[\begin{array}{c}
0 \\
0 \\
0 \\
\xi_{14} \\
\xi_{15}
\end{array}\right]+\underbrace{\left[\begin{array}{c}
H_{111}\left(t, x_{1}\right)+H_{121}\left(t, x_{2}\right) \\
0 \\
0 \\
H_{114}\left(t, x_{1}\right)+H_{124}\left(t, x_{2}\right) \\
0
\end{array}\right]}_{\xi_{1}\left(t, x_{1}\right)} \\
\dot{x}_{2}=\left[\begin{array}{c}
0.1 \sin x_{23}-x_{21} \\
-x_{21}^{2}-3 x_{22} \\
x_{21}^{2} x_{22}
\end{array}\right]+\left[\begin{array}{l}
0 \\
0 \\
1
\end{array}\right]\left(u_{1 j}+\phi_{2}\left(t, x_{2}\right)\right)+\underbrace{\left[\begin{array}{c}
0 \\
\xi_{22}\left(t, x_{2}\right) \\
0
\end{array}\right]}_{\sum_{j=1}}+\underbrace{\left[\begin{array}{c}
H_{212}\left(t, x_{1}\right)+H_{222}\left(t, x_{2}\right) \\
0
\end{array}\right]}_{\sum_{j=1}^{2} H_{2 j}\left(t, x_{j}\right)}
\end{gathered}
$$

where $x:=\operatorname{col}\left(x_{1}, x_{2}\right), x_{1}:=\operatorname{col}\left(x_{11}, x_{12}, x_{13}, x_{14}, x_{15}\right), x_{2}:=\operatorname{col}\left(x_{21}, x_{22}, x_{23}\right), u_{1} \in R^{2}, u_{2} \in R$ in the domain $x \in$ $\chi=\left\{\left(x_{1}, x_{2}\right)|| x_{15}-x_{12}^{2}\left|\leqslant \frac{1}{8},\right| x_{22}\left|\leqslant \frac{1}{2},\right| x_{23} \mid \leqslant 1\right\}$ and the uncertainties and interconnections satisfy

$$
\begin{gathered}
\left\|\phi_{1}\right\| \leqslant\left|\sin \left(x_{11}+x_{12}+x_{14}\right)\right|+\left|x_{13}\right| \\
\left\|\xi_{14}\right\| \leqslant 0.05 \sqrt{x_{12}^{2}+x_{14}^{2}}+0.05 \sqrt{x_{11}^{2}+x_{13}^{2}}+0.1\left\|x_{15}-x_{12}^{2}\right\|
\end{gathered}
$$




$$
\begin{gathered}
\left\|\xi_{15}\right\| \leqslant 0.1 \sqrt{x_{11}^{2}+x_{13}^{2}}+0.1\left\|x_{15}-x_{12}^{2}\right\| \\
\left\|H_{111}\left(t, x_{1}\right)+H_{121}\left(t, x_{2}\right)\right\| \leqslant\left|\sin \left(x_{13}\right)\right|\left\|x_{15}-x_{12}^{2}\right\|+0.5\left\|x_{21}\right\| \\
\left\|H_{114}\left(t, x_{1}\right)+H_{124}\left(t, x_{2}\right)\right\| \leqslant 0.49\left|x_{12}\right| \sqrt{x_{11}^{2}+x_{13}^{2}}+0.1\left\|x_{15}-x_{12}^{2}\right\|+0.1\left\|x_{21}\right\|+0.1\left\|x_{22}\right\| \\
\left\|\phi_{2}\left(t, x_{2}\right)\right\| \leqslant\left|\sin \left(x_{21}\right)\right|+\left|x_{22}\right| \\
\left\|\xi_{22}\left(t, x_{2}\right)\right\| \leqslant 0.1\left|x_{21}\right|+0.1\left|x_{22}\right| \\
\left\|H_{212}\left(t, x_{1}\right)+H_{222}\left(t, x_{2}\right)\right\| \leqslant 0.1 \sqrt{x_{11}^{2}+x_{13}^{2}}+0.1\left\|x_{15}-x_{12}^{2}\right\|+0.1\left|x_{21}\right|+0.1\left|x_{22}\right|
\end{gathered}
$$

Let

$$
h_{1}\left(x_{1}\right)=\left[\begin{array}{l}
h_{11}\left(x_{1}\right) \\
h_{12}\left(x_{1}\right)
\end{array}\right]=\left[\begin{array}{l}
x_{12} \\
x_{14}
\end{array}\right], \quad h_{2}\left(x_{2}\right)=x_{21}
$$

By direct computation, the nominal isolated subsystem of the first subsystem (40) has uniform relative degree $r_{1}=$ $\left(r_{11}, r_{12}\right)=(2,2)$, and the nominal isolated subsystem of the second subsystem (41) has relative degree $r_{2}=2$.

The coordinate transformation $z_{i}=T_{i}(x)$ for $i=1,2$ is given by

$$
\begin{gathered}
T_{1}=\left\{\begin{array}{c}
z_{1}^{a}=\left[\begin{array}{l}
z_{11} \\
z_{12} \\
z_{13} \\
z_{14}
\end{array}\right]=\left[\begin{array}{l}
x_{12} \\
x_{11} \\
x_{14} \\
x_{13}
\end{array}\right] \\
z_{1}^{b}=z_{15}=x_{15}-x_{12}^{2}
\end{array}\right. \\
T_{2}=\left\{\begin{array}{l}
z_{2}^{a}=\left[\begin{array}{l}
z_{21} \\
z_{22}
\end{array}\right]=\left[\begin{array}{c}
x_{21} \sin x_{23}
\end{array}\right] \\
-x_{21}+0.1 \log \\
z_{2}^{b}=z_{23}=x_{22}
\end{array}\right.
\end{gathered}
$$

Then consider the state feedback

$$
u_{i}=-\Lambda_{i}^{-1}\left(z_{i}\right) \alpha_{i}\left(z_{i}\right)+\Lambda_{i}^{-1}\left(z_{i}\right) \nu_{i} \quad i=1,2
$$

where

$$
\begin{gathered}
\Lambda_{1}=\left[\begin{array}{cc}
1 & 0 \\
0 & 2+\sin \left(z_{15}+z_{11}^{2}\right)
\end{array}\right], \quad \Lambda_{2}=0.1 \cos \left\{\arcsin \left\{10\left(z_{21}+z_{22}\right)\right\}\right\} \\
\alpha_{1}=\left[\begin{array}{c}
-\sin z_{11} \\
z_{11}^{2}+z_{14}^{2}
\end{array}\right], \quad \alpha_{2}=-z_{22}+0.1 z_{21}^{2} z_{23} \cos \left\{\arcsin \left\{10\left(z_{21}+z_{22}\right)\right\}\right\}
\end{gathered}
$$

In the new coordinates $z$ given in (50)-(51), by applying (52) to the nonlinear interconnected system (40)-(41), the closed-loop system is described by:

$$
\begin{aligned}
& \dot{z}_{11}^{a}=A_{1} z_{11}^{a}+B_{1} z_{12}^{a}+\Xi_{11}^{a}\left(t, z_{1}\right)+\Psi_{11}^{a}(t, z) \\
& \dot{z}_{12}^{a}=\nu_{1}+\Xi_{12}^{a}\left(t, z_{1}\right)+\Psi_{12}^{a}(t, z) \\
& \dot{z}_{1}^{b}=-4\left(z_{15}+z_{11}^{2}\right)+\Xi_{1}^{b}\left(t, z_{1}\right) \\
& \quad \dot{z}_{21}^{a}=A_{2} z_{21}^{a}+B_{2} z_{22}^{a} \\
& \dot{z}_{22}^{a}=\nu_{2}+\Xi_{22}^{a}\left(t, z_{2}\right) \\
& \dot{z}_{2}^{b}=-3 z_{23}-z_{21}^{2}+\Xi_{2}^{b}\left(t, z_{2}\right)+\Psi_{2}^{b}(t, z)
\end{aligned}
$$

where $z:=\operatorname{col}\left(z_{1}, z_{2}\right) \in \mathrm{Z}=\left\{\left(z_{1}, z_{2}\right)|| z_{15}\left|\leqslant \frac{1}{8},\right| \arcsin \left\{10\left(z_{21}+z_{22}\right)\right\}|\leqslant 1,| z_{23} \mid \leqslant \frac{1}{2}\right\}, z_{1}:=\operatorname{col}\left(z_{11}^{a}, z_{1}^{b}, z_{12}^{a}\right), z_{11}^{a}:=$ 
$\operatorname{col}\left(z_{11}, z_{13}\right), z_{12}^{a}:=\operatorname{col}\left(z_{12}, z_{14}\right), z_{1}^{b}:=z_{15}, z_{2}:=\operatorname{col}\left(z_{21}^{a}, z_{2}^{b}, z_{22}^{a}\right), z_{21}^{a}:=z_{21}, z_{22}^{a}:=z_{22}, z_{2}^{b}:=z_{23}, A_{1}=\left[\begin{array}{cc}0 & 0 \\ 0 & 0\end{array}\right]$, $B_{1}=\left[\begin{array}{ll}1 & 0 \\ 0 & 1\end{array}\right], A_{2}=0$ and $B_{2}=1$. Meanwhile, the uncertainties and interconnections satisfy

$$
\begin{gathered}
\left\|\Xi_{11}^{a}\left(t, z_{1}\right)\right\| \leqslant \rho_{1}\left(z_{11}^{a}, z_{12}^{a}, z_{1}^{b}\right)=\underbrace{0.05}_{\chi_{11}}\left\|z_{11}^{a}\right\|+\underbrace{0.05}_{\beta_{11}}\left\|z_{12}^{a}\right\|+\underbrace{0.1}_{\gamma_{11}}\left\|z_{1}^{b}\right\| \\
\left\|\Psi_{11}^{a}(t, z)\right\| \leqslant \underbrace{0.49\left|z_{11}\right|}_{\beta_{113}}\left\|z_{12}^{a}\right\|+\underbrace{0.1}_{\gamma_{113}}\left\|z_{1}^{b}\right\|+\underbrace{0.1}_{\chi_{123}}\left\|z_{21}^{a}\right\|+\underbrace{0.1}_{\gamma_{123}}\left\|z_{2}^{b}\right\| \\
\left\|\Xi_{12}^{a}\left(t, z_{1}\right)\right\| \leqslant \mu_{1}=\left|\sin \left(z_{11}+z_{12}+z_{13}\right)\right|+\left|z_{14}\right| \\
\left\|\Psi_{12}^{a}(t, z)\right\| \leqslant \underbrace{\sin \left(z_{14}\right) \mid}_{\gamma_{114}}\left\|z_{1}^{b}\right\|+\underbrace{0.5}_{\alpha_{124}}\left\|z_{21}^{a}\right\| \\
\left\|\Xi_{1}^{b}\left(t, z_{1}\right)\right\| \leqslant \underbrace{0.1}_{\beta_{12}}\left\|z_{12}^{a}\right\|+\underbrace{0.1}_{\gamma_{12}}\left\|z_{1}^{b}\right\| \\
\left\|\Xi_{22}^{a}\left(t, z_{2}\right)\right\| \leqslant \mu_{2}=\left|\sin \left(z_{21}\right)\right|+\left|z_{23}\right| \\
\left\|\Xi_{2}^{b}\left(t, z_{2}\right)\right\| \leqslant \underbrace{0.1}_{\alpha_{22}}\left\|z_{21}^{a}\right\|+\underbrace{0.1}_{\gamma_{22}}\left\|z_{2}^{b}\right\| \\
\left\|\Psi_{2}^{b}(t, z)\right\| \leqslant \underbrace{0.1}_{\beta_{215}}\left\|z_{12}^{a}\right\|+\underbrace{0.1}_{\gamma_{215}}\left\|z_{1}^{b}\right\|+\underbrace{0.1}_{\alpha_{225}}\left\|z_{21}^{a}\right\|+\underbrace{0.1}_{\gamma_{225}}\left\|z_{2}^{b}\right\|
\end{gathered}
$$

Choose $K_{1}=I_{2}, K_{2}=I_{1}, Q_{1}=4 I_{2}, Q_{2}=4 I_{1}$ and

$$
P_{1}=\left[\begin{array}{ll}
2 & 0 \\
0 & 2
\end{array}\right], \quad P_{2}=2
$$

Then choose $V_{1}^{b}=z_{11}^{2}+z_{13}^{2}+z_{15}^{2}$ and $V_{2}^{b}=z_{21}^{2}+z_{23}^{2}$. By calculation, it follows that in the considered domain in $z \in \mathrm{Z}$, Assumption 2 is satisfied with

$$
\begin{aligned}
& c_{11}=1, c_{12}=1, c_{13}=1, c_{14}=1, c_{15}=1, c_{16}=8, c_{17}=2, c_{18}=2 \\
& c_{21}=1, c_{22}=1, c_{23}=1, c_{24}=1, c_{25}=1, c_{26}=6, c_{27}=2, c_{28}=2
\end{aligned}
$$

Then, when the sliding motion takes place, inequalities (22)-(23) are satisfied with $\varepsilon_{111}=0.49\left|z_{11}\right|, \varepsilon_{112}=\varepsilon_{121}=\varepsilon_{122}=$ $\varepsilon_{13}=\varepsilon_{14}=\varepsilon_{17}=\varepsilon_{18}=\varepsilon_{27}=\varepsilon_{28}=\varepsilon_{215}=\varepsilon_{216}=\varepsilon_{225}=\varepsilon_{226}=0.1, \varepsilon_{115}=\varepsilon_{116}=\varepsilon_{125}=\varepsilon_{126}=\varepsilon_{23}=\varepsilon_{24}=\varepsilon_{211}=$ $\varepsilon_{212}=\varepsilon_{221}=\varepsilon_{222}=0$.

By direct computation,

$$
M+M^{T}=\left[\begin{array}{cccc}
8.4-5.88\left|z_{11}\right| & -0.8 & -3.6-1.96\left|z_{11}\right| & -1.2 \\
-0.8 & 9.2 & -1.2 & -1.6 \\
-3.6-1.96\left|z_{11}\right| & -1.2 & 14.8 & -0.4 \\
-1.2 & -1.6 & -0.4 & 11.2
\end{array}\right]
$$

which is symmetric positive definite in $z \in \mathrm{Z}$.

Hence the conditions of Theorem 1 hold.

Based on the parameter selections above, the control (35) is well defined with $\eta_{1}=0.01, \eta_{2}=0.01$ and $\Phi_{1}, \Phi_{2}$ given by:

$$
\begin{gathered}
\Phi_{1}=0.49\left|z_{11}\right|\left\|z_{12}^{a}\right\|+\left(\left|\sin \left(z_{14}\right)\right|+0.1\right)\left\|z_{1}^{b}\right\| \\
\Phi_{2}=0.6\left\|z_{21}^{a}\right\|+0.1\left\|z_{2}^{b}\right\|
\end{gathered}
$$

For simulation purposes, $\sigma_{i} /\left(\left\|\sigma_{i}\right\|+\beta_{i}\right)$ is used to replace $\operatorname{sgn}\left(\sigma_{i}\right)$ in order to reduce the chattering with $\beta_{1}=\beta_{2}=0.001$. 
The time response of the system states is shown in Fig.1. The time response of the system control signals is shown in Fig.2. The time response of the sliding functions is shown in Fig.3. It can be seen that the subsystems are stabilized despite the presence of unmatched uncertainties $\xi_{i}$ and interconnections $\sum_{j=1}^{n} H_{i j}\left(t, x_{j}\right)$. The nonlinear interconnected system is driven to the sliding surface (18) in finite time. The simulation results show that the proposed decentralised sliding mode control is effective. The obtained results validate the approach of transforming system (40)-(41) into system (55)-(56) by local coordinate transformation and feedback linearization.

Remark 12: All the simulation parameters have been given. The $h_{i}\left(x_{i}\right)$ are chosen to impose a uniform relative degree $r_{i}$ based on [29]. The $\eta_{i}$ are small positive constants to guarantee the reachability condition (35) is satisfied. For convenience, $Q_{i}=\alpha_{i} I_{n_{i}}$ with the $\alpha_{i}$ positive constants. Then the $P_{i}$ are positive definite symmetric matrices to guarantee $M^{T}+M>0$ as defined in (24). Finally, $K_{i}$ can be obtained by solving the LMI (17) since $P_{i}$ and $Q_{i}$ have been chosen.

Remark 13: It should be noted that the above example has the following characteristics: (i) the nominal isolated systems are fully nonlinear; (ii) the uncertainties $\xi_{i}$ are nonlinear and cannot be bounded by a linear function of $x_{i}$; (iii) the interconnections $\sum_{j=1}^{n} H_{i j}\left(t, x_{j}\right)$ are also nonlinear and cannot be bounded by a linear function of $x_{j}$; (iv) the uncertainties $\xi_{i}$ and interconnections $\sum_{j=1}^{n} H_{i j}\left(t, x_{j}\right)$ are both unmatched. Therefore, existing decentralised state feedback schemes ([38], [39], [40], [41]) cannot be applied to the nonlinear interconnected system (40)-(41). Furthermore, the schemes of [27] cannot be applied unless the system (40)-(41) is in the required regular form. This shows the value of the proposed results.

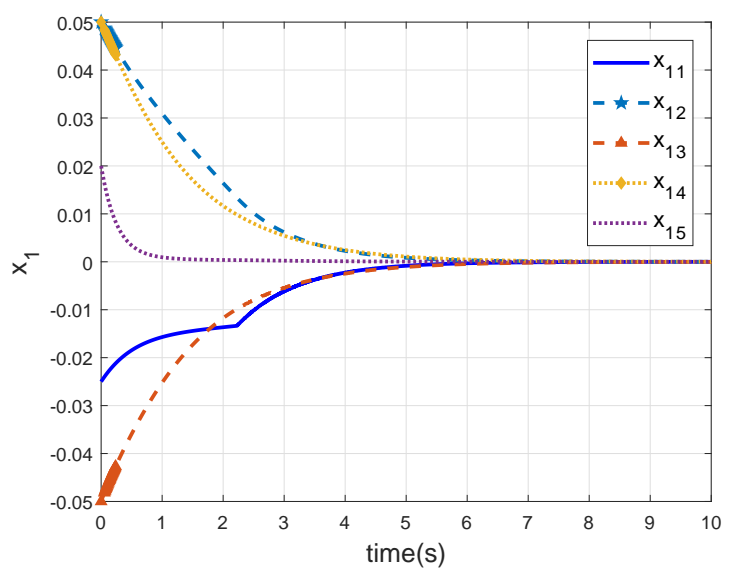

(a) The states of the first subsystem

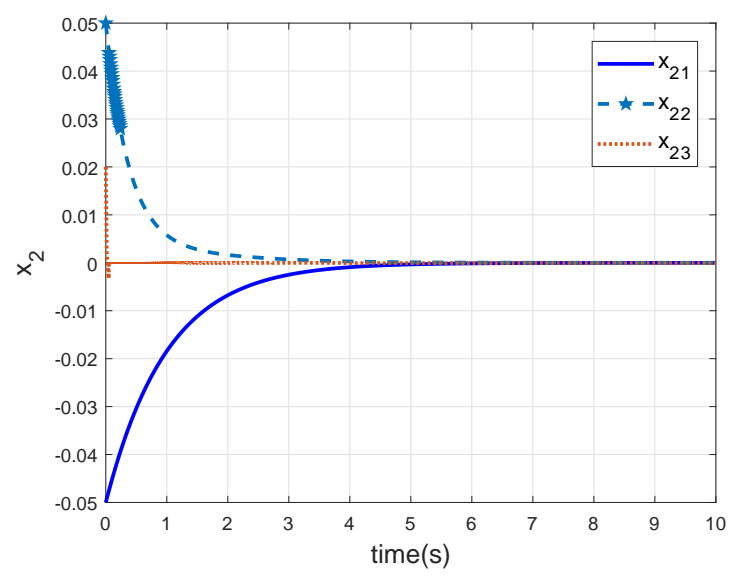

(b) The states of the second subsystem

Fig. 1: The time response of the system states

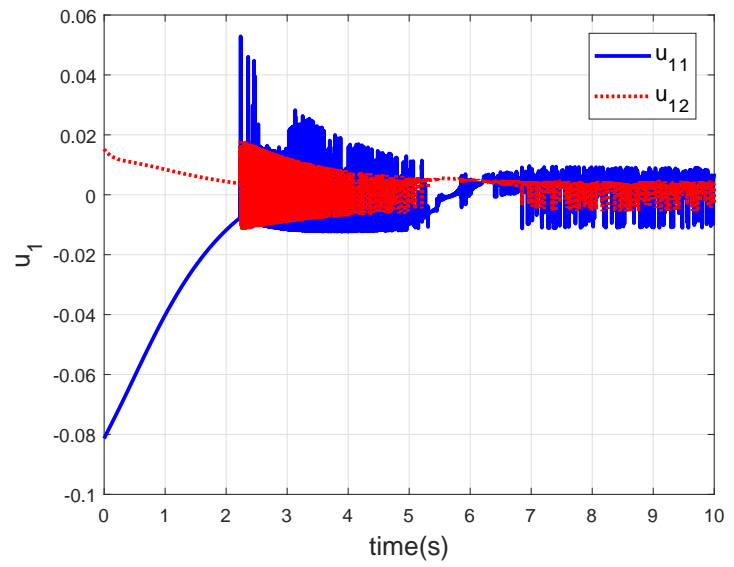

(a) The control signals of the first subsystem

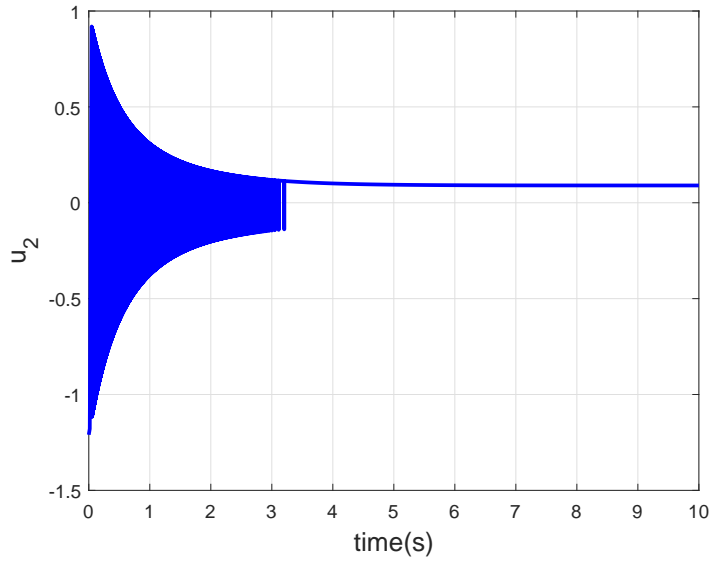

(b) The control signals of the second subsystem

Fig. 2: The time response of the system control signals 


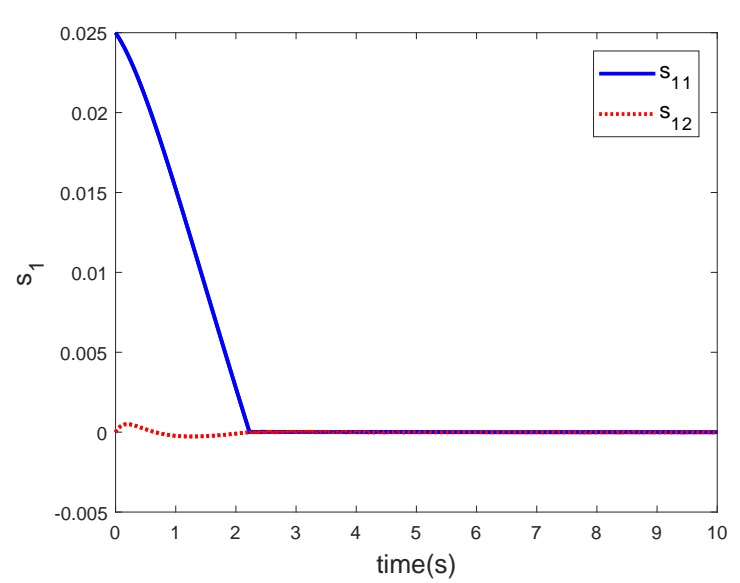

(a) The sliding functions of the first subsystem

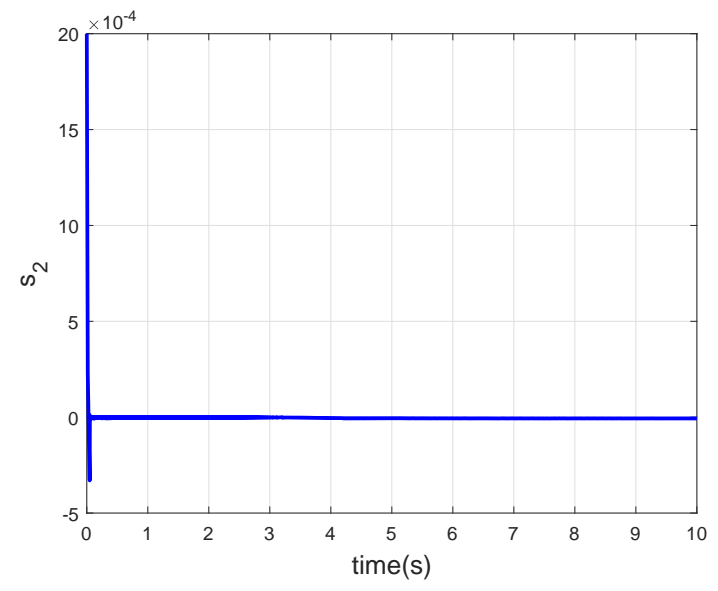

(b) The sliding functions of the second subsystem

Fig. 3: The time response of the sliding functions

To further test the proposed decentralised sliding mode control, the results obtained using the decentralised state feedback control (DSFC) scheme proposed in [23] will be compared with the proposed method. The main control parameters in [23] are given:

$$
\begin{array}{cc}
\tilde{K}_{1}=\left[\begin{array}{cccc}
10 & 20 & -5 & -3 \\
2 & 10 & 2 & 3
\end{array}\right], \tilde{P}_{1}=\left[\begin{array}{cccc}
2.27 & 0.02 & 0.10 & 0.14 \\
0.02 & 0.05 & -0.04 & -0.06 \\
0.10 & -0.04 & 1.14 & 0.14 \\
0.14 & -0.06 & 0.14 & 0.16
\end{array}\right] \\
\tilde{K}_{2}=\left[\begin{array}{cc}
1 & 1
\end{array}\right], & \tilde{P}_{2}=\left[\begin{array}{cc}
1.50 & 0.50 \\
0.50 & 1.00
\end{array}\right]
\end{array}
$$

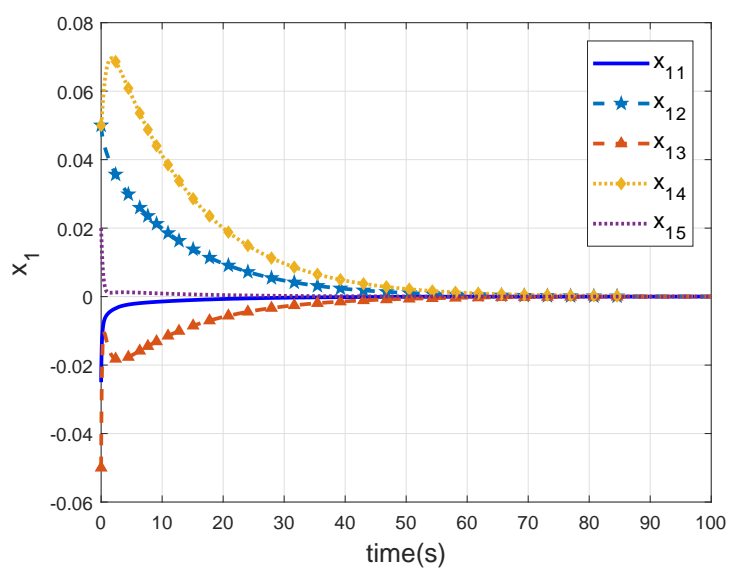

(a) The states of the first subsystem

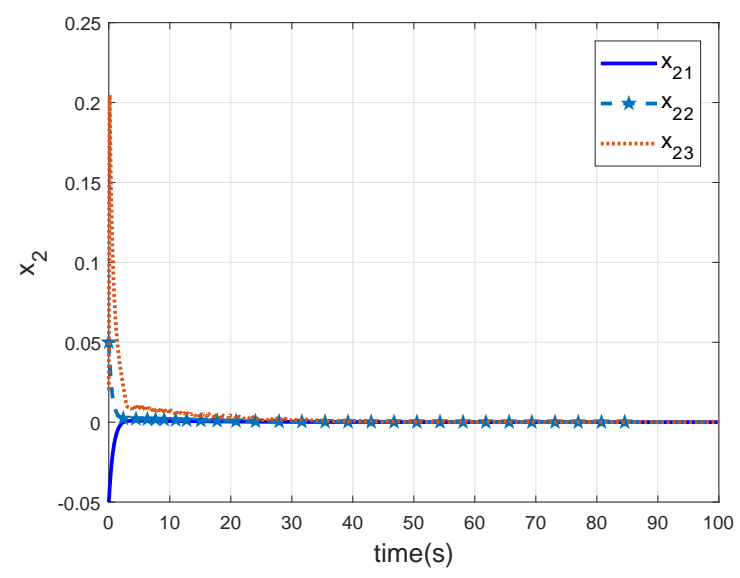

(b) The states of the second subsystem

Fig. 4: The time response of the system states by using DSFC proposed in [23] 


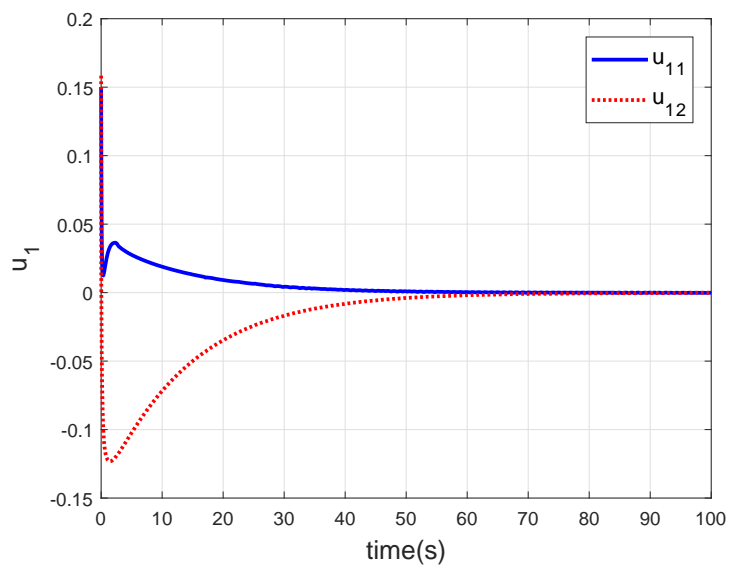

(a) The control signals of the first subsystem

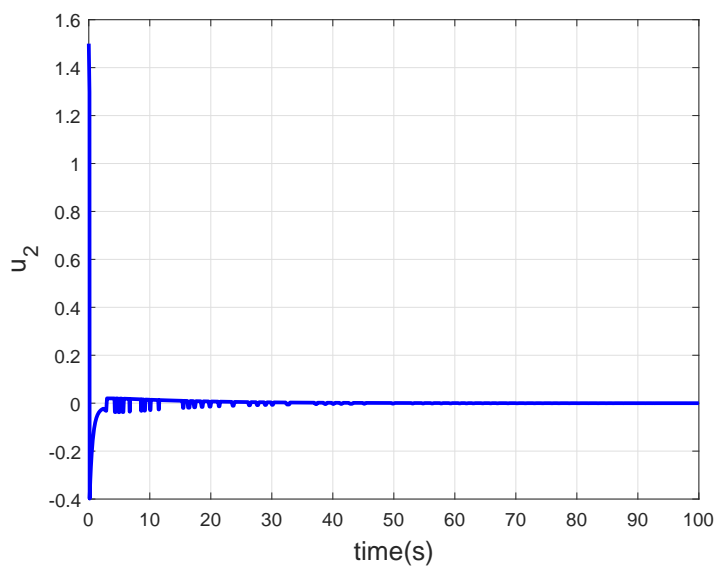

(b) The control signals of the second subsystem

Fig. 5: The time response of the system control signals by using DSFC proposed in [23]

The time response of the system states using the DSFC proposed in [23] is shown in Fig.4. The corresponding time response of the system control signals is shown in Fig.5. It should be noted that the unmatched interconnections $\Psi_{11}^{a}(t, z)$ have not been considered in [23], but the method proposed in this paper can accommodate the unmatched interconnections. Meanwhile, the unmatched uncertainties $\Xi_{11}^{a}\left(t, z_{1}\right)$ are required to satisfy a weakly unmatched condition in [23]; stronger unmatched uncertainties can be dealt with in this paper. Comparing Fig.1 with Fig.4, the proposed approach has a faster response speed due to its direct consideration of the unmatched uncertainties and the unmatched interconnections.

In order to further test the robustness of the proposed decentralised sliding mode control, unmatched uncertainties $\xi_{14}$ and interconnections $H_{114}\left(t, x_{1}\right)+H_{124}\left(t, x_{2}\right)$ of the system (40)-(41) are introduced, while the other terms remain unchanged. Specifically, the conditions (43) and (46) become:

$$
\begin{gathered}
\left\|\xi_{14}\right\| \leqslant 0.2 \sqrt{x_{12}^{2}+x_{14}^{2}}+0.2 \sqrt{x_{11}^{2}+x_{13}^{2}}+0.4\left\|x_{15}-x_{12}^{2}\right\| \\
\left\|H_{114}\left(t, x_{1}\right)+H_{124}\left(t, x_{2}\right)\right\| \leqslant 1.96\left|x_{12}\right| \sqrt{x_{11}^{2}+x_{13}^{2}}+0.4\left\|x_{15}-x_{12}^{2}\right\|+0.4\left\|x_{21}\right\|+0.4\left\|x_{22}\right\|
\end{gathered}
$$

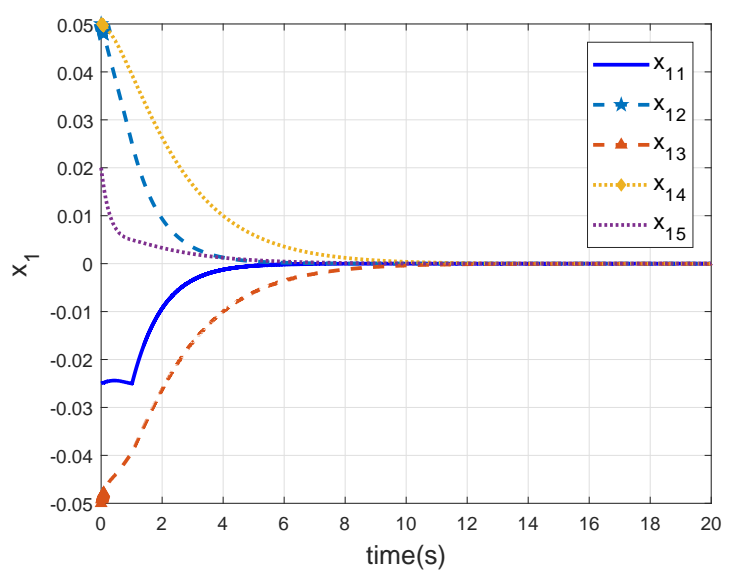

(a) The states of the first subsystem

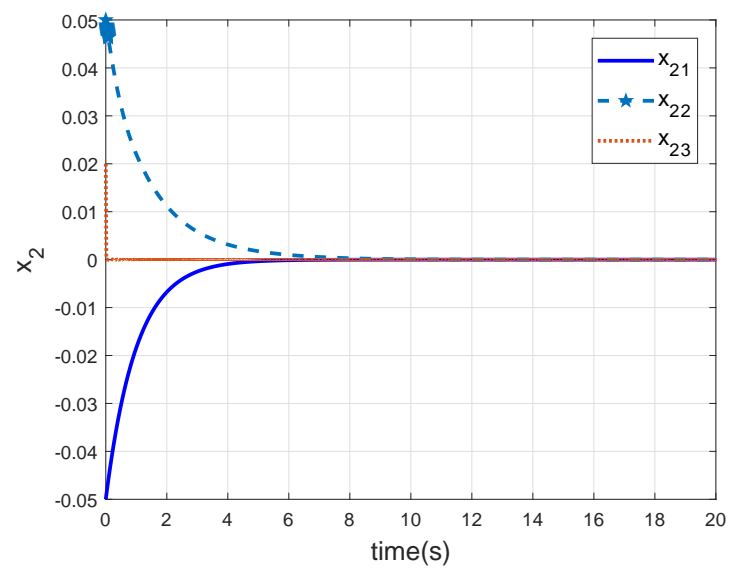

(b) The states of the second subsystem

Fig. 6: The time response of the system states by using the method proposed in this paper under the new conditions (71)-(72) 


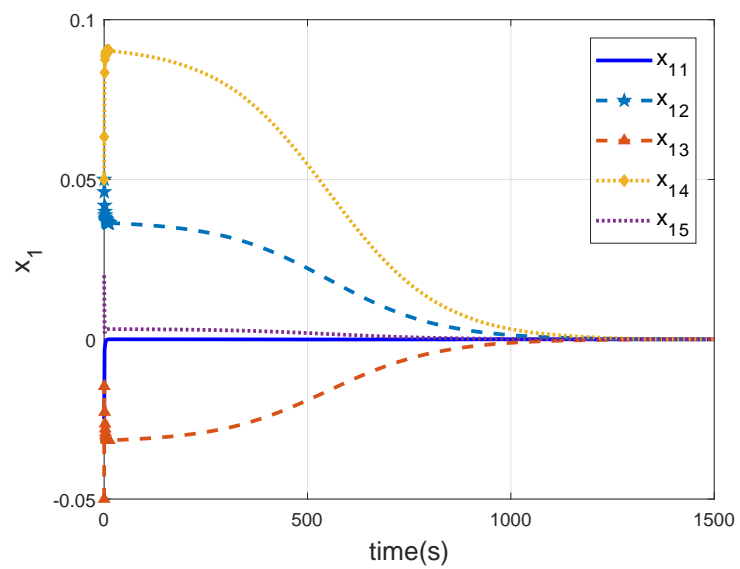

(a) The states of the first subsystem

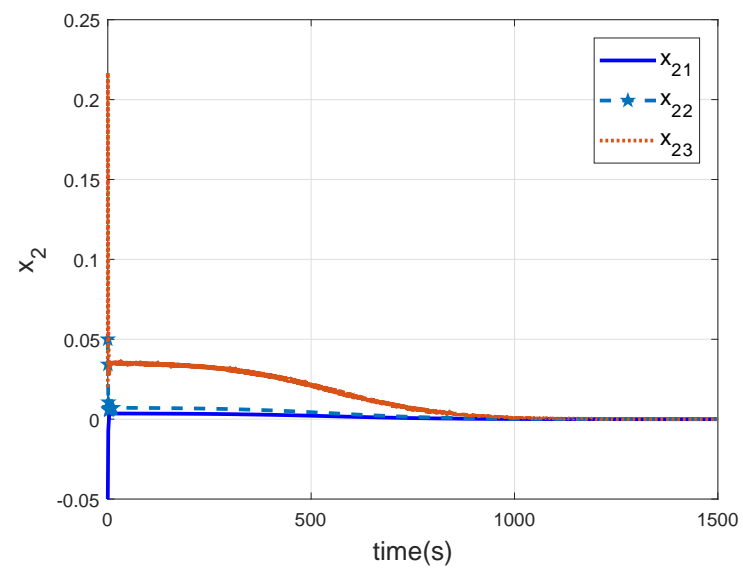

(b) The states of the second subsystem

Fig. 7: The time response of the system states by using DSFC proposed in [23] under the new conditions (71)-(72)

The time response of the system states under the new conditions (71)-(72) using the method proposed in this paper are shown in Fig.6. The corresponding time response of the system states using the DSFC proposed in [23] is shown in Fig.7. Comparing Fig.6 with Fig.7, it can be seen that although the unmatched interconnections and uncertainties increase, the proposed method achieves better performance.

\section{CONCLUSION}

A robust decentralized control design method has been proposed for a class of interconnected large-scale systems with nonlinear nominal isolated systems. The uncertainties and interconnections of the systems are all nonlinear with nonlinear bounds. The bounds on the uncertainties and interconnections are assumed to be known functions. These have been employed to reject the effects of the uncertainty and interconnections on the system to enhance robustness. A sliding mode control has been designed to ensure that the system states reach the designed sliding surface in finite time. The obtained results can be applied to a class of interconnected systems with uniform relative degree and involutive distribution. A numerical example is given to show the effectiveness of the proposed decentralised control scheme. Future work will focus on experimental testing and the practical application of the proposed method. From the theoretical point of view, the problem of decentralized output feedback sliding mode control will be considered within the same design framework.

\section{DECLARATION OF CONFLICTING INTERESTS}

The author(s) declared no potential conflicts of interest with respect to the research, authorship, and/or publication of this article.

\section{FUNDING}

This work is partially supported by the National Nature Science Foundation of China under grant nos 61973315 and the Thousand Talents Program for foreign experts.

\section{REFERENCES}

[1] M. Cucuzzella, G. P. Incremona, A. Ferrara, Decentralized sliding mode control of islanded ac microgrids with arbitrary topology, IEEE Transactions on Industrial Electronics 64 (8) (2017) 6706-6713.

[2] A. Som, D. Das, Seismic vibration control of offshore jacket platforms using decentralized sliding mode algorithm, Ocean Engineering 152 (2018) 377-390.

[3] P. Li, C. Zhang, X. Fu, G. Song, C. Wang, J. Wu, Determination of local voltage control strategy of distributed generators in active distribution networks based on kriging metamodel, IEEE Access 7 (2019) 34438-34450.

[4] L. Bakule, Decentralized control: An overview, Annual reviews in control 32 (1) (2008) 87-98.

[5] A. Bonfiglio, F. Cantoni, A. Oliveri, R. Procopio, A. Rosini, M. Invernizzi, M. Storace, An mpc-based approach for emergency control ensuring transient stability in power grids with steam plants, IEEE Transactions on Industrial Electronics 66 (7) (2019) 5412-5422. 
[6] J. Chen, Q. Song, A decentralized energy management strategy for a fuel cell/supercapacitor-based auxiliary power unit of a more electric aircraft, IEEE Transactions on Industrial Electronics 66 (7) (2019) 5736-5747.

[7] Z. Gu, P. Shi, D. Yue, Z. Ding, Decentralized adaptive event-triggered $h_{\infty}$ filtering for a class of networked nonlinear interconnected systems, IEEE Transactions on Cybernetics 49 (5) (2019) 1570-1579.

[8] P. Du, H. Liang, S. Zhao, C. K. Ahn, Neural-based decentralized adaptive finite-time control for nonlinear large-scale systems with time-varying output constraints, IEEE Transactions on Systems, Man, and Cybernetics: Systems (2019) 1-12.

[9] X.-G. Yan, S. K. Spurgeon, C. Edwards, Decentralised sliding mode control for nonminimum phase interconnected systems based on a reduced-order compensator, Automatica 42 (10) (2006) 1821-1828.

[10] X. Li, B. Zhang, P. Li, Q. Zhou, R. Lu, Finite-horizon h-infinity state estimation for periodic neural networks over fading channels, IEEE Transactions on Neural Networks and Learning Systems (2019) 1-11.

[11] L. Zhang, H. Lam, Y. Sun, H. Liang, Fault detection for fuzzy semi-markov jump systems based on interval type-2 fuzzy approach, IEEE Transactions on Fuzzy Systems (2019) 1-1.

[12] H. Bevrani, M. Abrishamchian, N. Safari-Shad, Nonlinear and linear robust control of switching power converters, in: Proceedings of the 1999 IEEE International Conference on Control Applications (Cat. No. 99CH36328), Vol. 1, IEEE, 1999, pp. 808-813.

[13] S. Ray, G. K. Venayagamoorthy, B. Chaudhuri, R. Majumder, Comparison of adaptive critic-based and classical wide-area controllers for power systems, IEEE Transactions on Systems, Man, and Cybernetics, Part B (Cybernetics) 38 (4) (2008) 1002-1007.

[14] J. Mu, X.-G. Yan, S. K. Spurgeon, Z. Mao, Generalized regular form based smc for nonlinear systems with application to a wmr, IEEE Transactions on Industrial Electronics 64 (8) (2017) 6714-6723.

[15] Y. Mi, Y. Fu, C. Wang, P. Wang, Decentralized sliding mode load frequency control for multi-area power systems, IEEE Transactions on Power Systems 28 (4) (2013) 4301-4309.

[16] C.-C. Cheng, Y. Chang, Design of decentralised adaptive sliding mode controllers for large-scale systems with mismatched perturbations, International Journal of Control 81 (10) (2008) 1507-1518.

[17] J. Lee, On the decentralized stabilization of interconnected variable structure systems using output feedback, Journal of the Franklin Institute 332 (5) (1995) 595-605.

[18] X.-G. Yan, C. Edwards, S. K. Spurgeon, Decentralised robust sliding mode control for a class of nonlinear interconnected systems by static output feedback, Automatica 40 (4) (2004) 613-620.

[19] X.-G. Yan, S. K. Spurgeon, C. Edwards, Memoryless static output feedback sliding mode control for nonlinear systems with delayed disturbances, IEEE Transactions on Automatic Control 59 (7) (2014) 1906-1912.

[20] X.-G. Yan, S. K. Spurgeon, C. Edwards, Decentralised stabilisation for nonlinear time delay interconnected systems using static output feedback, Automatica 49 (2) (2013) 633-641.

[21] X.-J. Li, X.-X. Ren, G.-H. Yang, Backstepping-based decentralized tracking control for a class of interconnected stochastic nonlinear systems coupled via a directed graph, Information Sciences 477 (2019) 302-320.

[22] C.-C. Hua, X.-P. Guan, Smooth dynamic output feedback control for multiple time-delay systems with nonlinear uncertainties, Automatica 68 (2016) $1-8$.

[23] X.-G. Yan, S. K. Spurgeon, D. Zhao, L. Guo, Decentralised stabilisation of nonlinear time delay interconnected systems, IFAC-PapersOnLine 49 (9) (2016) 152-157.

[24] J. Sun, C. Liu, Decentralised zero-sum differential game for a class of large-scale interconnected systems via adaptive dynamic programming, International Journal of Control 92 (12) (2019) 2917-2927.

[25] Y.-Q. Han, H.-S. Yan, Observer-based multi-dimensional taylor network decentralised adaptive tracking control of large-scale stochastic nonlinear systems, International Journal of Control (2018) 1-14.

[26] C. Edwards, S. Spurgeon, Sliding mode control: theory and applications, Crc Press, 1998.

[27] J. Mu, X.-G. Yan, S. K. Spurgeon, Z. Mao, Decentralised sliding mode control for nonlinear interconnected systems in the regular form, in: 2016 IEEE 55th Conference on Decision and Control (CDC), IEEE, 2016, pp. 6452-6457.

[28] X.-G. Yan, S. K. Spurgeon, Delay-independent decentralised output feedback control for large-scale systems with nonlinear interconnections, International Journal of Control 87 (3) (2014) 473-482.

[29] A. Isidori, Nonlinear control systems, Springer Science \& Business Media, 2013.

[30] R. B. Gardner, W. F. Shadwick, The gs algorithm for exact linearization to brunovsky normal form, IEEE Transactions on Automatic Control 37 (2) (1992) 224-230.

[31] P. Gahinet, A. Nemirovskii, A. J. Laub, M. Chilali, The 1mi control toolbox, in: Proceedings of 1994 33rd IEEE Conference on Decision and Control, Vol. 3, IEEE, 1994, pp. 2038-2041.

[32] Q. Liu, H. S. Abbas, J. M. Velni, An lmi-based approach to distributed model predictive control design for spatially-interconnected systems, Automatica 95 (2018) 481-487.

[33] H. K. Khalil, J. W. Grizzle, Nonlinear systems, Prentice hall Upper Saddle River, NJ, 2002.

[34] J.-J. E. Slotine, W. Li, et al., Applied nonlinear control, Prentice hall Englewood Cliffs, NJ, 1991.

[35] K.-C. Hsu, Decentralized variable-structure control design for uncertain large-scale systems with series nonlinearities, International Journal of Control 68 (6) (1997) 1231-1240.

[36] Y. Fujisaki, R. Sakuwa, Estimation of asymptotic stability regions via homogeneous polynomial lyapunov functions, International Journal of Control 79 (06) (2006) 617-623.

[37] K. Ichikawa, R. Ortega, On stabilization of nonlinear systems with enlarged domain of attraction, Automatica 28 (3) (1992) $623-626$.

[38] L. Zhu, Y. Lv, Decentralised controller design for a class of interconnected systems, The Journal of Engineering 2019 (15) (2019) $593-598$.

[39] Z. Chen, J. Tan, X. Wang, Z. Cao, Decentralized observer-based 12-1 tracking control for a class of interconnected markovian jump system, in: 2018 Ninth International Conference on Intelligent Control and Information Processing (ICICIP), 2018, pp. 16-20.

[40] A. E. Onyeka, X. Yan, Z. Mao, Q. Zhang, Decentralized sliding mode lfc for nonlinear interconnected power system with time delay, in: 2018 Annual American Control Conference (ACC), 2018, pp. 6666-6671.

[41] L. Zhang, C. Hua, G. Cheng, K. Li, X. Guan, Decentralized adaptive output feedback fault detection and control for uncertain nonlinear interconnected systems, IEEE Transactions on Cybernetics (2018) 1-11. 